\title{
ANÁLISE DE ENSAIOS EM PARCELAS SUBDIVIDIDAS COM OBSERVAÇÕES PERDIDAS
}

VALDENIR QUEIROZ RIBEIRO

Engentedro-aurônomo

Orientador: Dr. Izaias Rangel Nogueira

Disserłação apresentada à Escola Superior de Agriculłura "Luiz de Queiroz", da Universidade de São Paulo, para obłenção do título de Mestre em Esłałística e Experimentação Agronômica.

\author{
$P|R A C| C A B A$
}

Estado de Säo Paulo - Brasil

Março, 1980 
ii.

A meus pais Vicente e Corina

A meus sogros Luis e Berenice

À minha esposa Maria de Jesus

A meus filhos Vladimir e Patricia

$D E D I C O$. 


\section{AGRADECIMENTOS}

Ao Professor Dr. Izaias Rangel Nogueira, Chefe do De partamento de Matemática e Estatística da Escola Superior de Agricultura "Luiz de Queiroz", pela orientação segura na realização des te trabalho.

A Empresa Brasileira de Pesquisa Agropecuäria (EMBRA PA), pela oportunidade oferecida para a realização do Curso de Estatística e Experimentação Agronómica.

Ao Professor Dr. F. Pimentel Gomes pelo seu valor hu mano e dedicação ao Curso de Pōs-Graduação.

Ao Professor Dr. Décio Barbin, pelo apoio dispensado.

Ao Professor Dr. Humberto de Campos pelas valiosas sugestões na elaboração deste trabalho.

Ao Professor Dr. Vivaldo Francisco da Cruz pela versão do resumo para o inglês.

Aos demais Professores do Departamento de Matemática e Estatística da Escola Superior de Agricultura "Luiz de Queiroz", pelos ensinamentos recebidos e proveitosos diálogos.

A colega Clarice Garcia Borges Demétrio pela revisão da redação.

Aos colegas: Humberto Coelho, José Fernando Soares Dias, Francisco das Chagas Estevam da Fonseca e Alexandre Reinaldo da Costa Lima, pela amizade e troca de idéias durante o Curso.

Aos demais colegas do Curso de Pós-Graduação, pelo excelente convívio durante o Curso.

Ao Eng ${ }^{8}-A g r^{8}$ Antonio Milton de Araújo Rocha, pertencente à Delegacia Estadual do Ministério de Agricultura - DEMA (PI), pelo apoio dispensado e amizade.

Aos funcionários do Departamento de Matemática e Estatística: Maria Izalina Ferreira Alves, Rosa Maria Alves, Ôctávio Frasseto e Maria Alayde Penteado de Souza, pela amizade e atenção dispensada. 


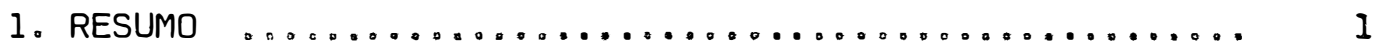

2. INTRODUÇÃO $\ldots \ldots \ldots \ldots \ldots \ldots \ldots \ldots \ldots \ldots \ldots \ldots \ldots \ldots \ldots \ldots \ldots \ldots \ldots$

3. REVISÃO DE LITERATURA $\ldots \ldots \ldots \ldots \ldots \ldots \ldots \ldots \ldots \ldots \ldots \ldots \ldots \ldots \ldots \ldots \ldots \ldots \ldots$

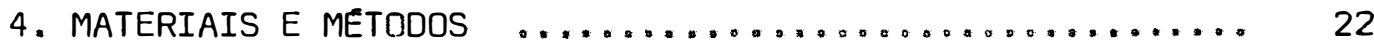

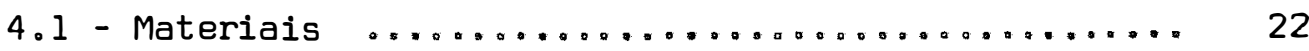

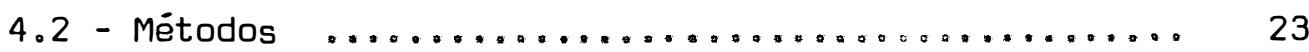

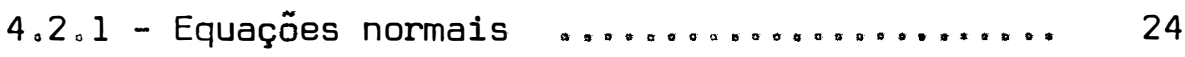

4.2 .2 - Estimativas dos efeitos dos parâmetros .. 25

4.2 .3 - Estimativas das subparcelas perdidas .... 26

4.2 .4 - Obtenção das somas de quadrados ajustadas 28

4.2.5 - Cálculo das correções "U" para a soma de quadrados da interação TxT' $\ldots \ldots \ldots \ldots . .34$

4.2 .6 - Análise de variância $\ldots \ldots \ldots \ldots \ldots \ldots . . \ldots \ldots$

4.2.7 - Estimativas de variâncias das estimativas de contrastes entre duas médias de trata-

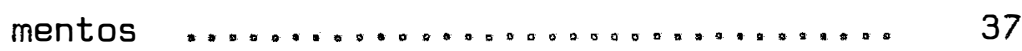

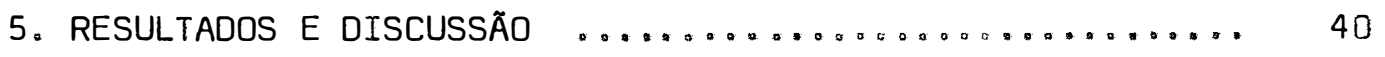

5.1 - Caso de Uma Subparcela Perdida .............. 41

5.2 - Caso de Duas Subparcelas Perdidas Numa Mesma Parce la Composta de $k>2$ Subparcelas $\ldots \ldots \ldots \ldots \ldots . . . .44$

5.3 - Caso de Duas Subparcelas Perdidas que Receberam Tra tamentos $T$ e $T$ ' Diferentes .................... 48 
5.4 - Caso de Duas Subparcelas Perdidas que Receberam Tra tamentos $T$ Diferentes e Mesmo Tratamento $T$ ' .....

5.5 - Caso de Duas Subparcelas Perdidas que Receberam Mes mo Tratamento $T$ e Tratamentos T' Diferentes, em Par celas Distintas $\ldots \ldots \ldots \ldots \ldots \ldots \ldots \ldots$

5.6 - Caso de Duas Subparcelas Perdidas que Receberam Mes mos Tratamentos $T$ e $T^{\prime} \ldots \ldots \ldots \ldots \ldots \ldots \ldots \ldots \ldots \ldots \ldots \ldots \ldots$

5.7 - Caso de Uma Parcela Perdida, Composta de Duas Sub-

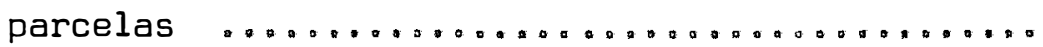

5.8 - Caso de Uma Parcela Perdida, Composta de k Subparcelas

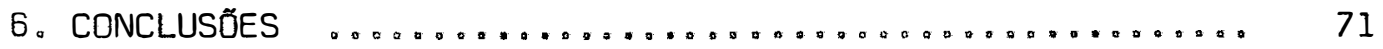

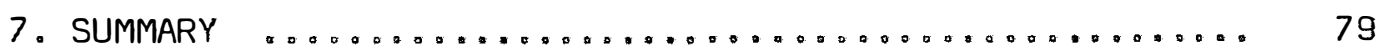

8. LITERATURA CITADA $\ldots \ldots \ldots \ldots \ldots \ldots \ldots \ldots \ldots \ldots \ldots \ldots \ldots$

9. APENDICE $\ldots \ldots \ldots \ldots \ldots \ldots \ldots \ldots \ldots \ldots \ldots \ldots \ldots \ldots \ldots \ldots \ldots \ldots \ldots \ldots$ 


\section{RESUMO}

O presente trabalho teve como objetivo determinar fór mulas para estimativas de subparcelas perdidas, correções "U" para as somas de quadrados da interação $T \times T^{\prime}$ e estimativas das variâncias das estimativas de contrastes entre duas mëdias de tratamentos, em delineamento inteiramente casualizado com parcelas subdivididas. Foram considerados os casos onde se perderam uma e duas subparcelas e uma parcela constituída de $k \geq 2$ subparcelas. As estimativas das subparcelas foram determinadas uti lizando-se da minimizaçäo da soma de quadrados do resíduo (b),

$$
[\mathrm{SQR}(b)] \text {. }
$$

As expressöes das correções "U" foram determinadas através do método do resíduo condicional de R.A. Fisher. 
As somas de quadrados da interação TxT' ajustadas foram obtidas pelo método do resíduo condicional e pela utilizaçäo das correções "U".

Foi demonstrado, para os casos de uma e duas subparcelas perdidas, que a soma de quadrados de tratamentos T' ajustada $\left[S Q T^{\prime}(a j)\right]$, pode ser obtida pela seguinte expressão:

$$
\begin{gathered}
\operatorname{SQT}^{\prime}(a j)=\operatorname{SQTotal}(\text { usual) } \\
-\operatorname{SQParcelas}(i j)\left(\text { usual) }-\operatorname{SQT} \times T^{\prime}(a j)-\right. \\
-\operatorname{SQR}(b)
\end{gathered}
$$

Partindo-se das funções lineares de contrastes entre duas médias de tratamento foram determinadas suas respectivas esti mativas de variàncias.

Para o caso onde foi perdida uma parcela constitulda de $k \geq 2$ subparcelas, foi verificado que as somas de quadrados da in teração TXT' ajustadas e de tratamentos $T^{\prime}$ ajustadas podem ser determinadas de maneira usual, isto é, sem incluir as estimativas das subparcelas perdidas. 


\section{INTRODUÇÃO}

Apesar de todo o cuidado dispensado ao planejamento e à condução de uma pesquisa, a ocorrência de perdas de parcelas é co mum, em trabalhos experimentais, e a análise estatistica dos dados se torna mais complicada.

0 conceito estatistico de parcela perdida inclui tam bém aquelas que apresentam dados de observações discrepantes dos de mais, e cuja causa não é atribuída ao efeito de tratamentos estuda dos.

$$
\text { Para se efetuar a análise de variãncia em ensaios }
$$
com parcelas perdidas, um dos procedimentos é determinar estimativas dos valores perdidos e colocá-las nos lugares das parcelas perdidas e em seguida, proceder à análise como se todos os dados fossem conhecidos. 
As estimativas dos valores perdidos não substituem os seus verdadeiros valores, que săo desconhecidos, o que acarreta uma anälise de variância com vícios ("bias") das somas de quadrados, necessitando, portanto, uma correção das mesmas. Neste trabalho propõem-se fórmulas para estimativas de subparcelas perdidas em delineamento inteiramente casualizado com parcelas subdivididas, nos casos onde foram perdidas uma e duas sub parcelas e uma parcela constituída de $k \geq 2$ subparcelas.

Além das fórmulas para as estimativas das subparcelas perdidas, foram determinadas as correções "U" para as somas de quadrados e as estimativas de variâncias das estimativas de contras tes entre médias de tratamentos ajustados. 
5.

\section{REVISAO DE LITERATURA}

As anälises estatisticas de ensaios com estimativas de parcelas perdidas, apresentam várias fases: estimativas das parcelas perdidas sob um aspecto matemático rigoroso, análise de variância de maneira usual, correções das somas de quadrados super ou subestimadas e cálculos das estimativas de variâncias das estimativas de contrastes entre médias de tratamentos ajustados.

ALLAN e WISHART (1930) foram os primeiros autores a se preocuparem com a determinaçăo de estimativas de parcelas perdidas, e conceituaram essas parcelas como aquelas cujas observaçōes são imperfeitas, devido a causas além do controle do próprio experí mentador.

ANDERSON (1946) definiu parcelas perdidas de maneira anāloga a ALLAN e WISHART (1930), e recomendou que sejam rejeitadas 
somente em casos extremos, quando comprovado que o tratamento em questão não ẻ responsável pelas aparentes anomalias dos resultados. Este autor apresentou fórmulas para cálculos de subparcelas perdidas em ensaios com parcelas subdivididas, pela minimi zação da variância residual.

o mesmo autor aplicou o método da covariância em um experimento com $\underline{\underline{I}}$ repetiçōes, $\underline{\alpha}$ parcelas e $\underline{\beta}$ subparcelas, onde a sub parcela perdida recebeu os tratamentos $a_{1}, b_{1}$, na repetição $r_{1}$, e estabeleceu como a melhor estimativa da subparcela perdida, a fim de minimizar o erro da subparcela, o residuo do coeficiente $\underline{b}$ de re gressäo:

$$
y=\frac{r\left(R_{1} A_{1}\right)+B\left(A_{1} B_{1}\right)-A_{1}}{(r-1)(B-1)},
$$

onde, y: estimativa da subparcela perdida;

$A_{1}$ : total das observaçōes com o tratamento $a_{1}$;

$\left(R_{1} A_{1}\right)$ : total das observações existentes com o tratamento $a_{1}$ na re petição $r_{1}$ :

$\left(A_{1} B_{1}\right)$ : total das observaçöes com os tratamentos $a_{1}$ e $b_{1}$.

PINHO (1973), utilizando o método da soma mínima de quadrados apresentado por YATES (1933), determinou fórmulas para es timativas de uma e duas subparcelas perdidas em um experimento em parcelas subdivididas com a tratamentos $A$ aplicados às parcelas, dis postos em $\underline{I}$ blocos casualizados, ㄴ tratamentos B aplicados às sub- 
parcelas, nos seguintes casos:

a) Uma subparcela perdida:

$$
y=\frac{r P+b\left(A_{i} B_{k}\right)-A_{i}}{(r-1)(b-1)} .
$$

A autora verificou que esta fórmula colncide com aque la determinada por ANDERSON (1946), o qual utilizou o método da covariāncia.

b) Duas subparcelas perdidas numa mesma parcela:

$$
\begin{aligned}
& x=\frac{r P+(b-1)\left(A_{i} B_{k 1}\right)+\left(A_{i} B_{k 2}\right)-A_{i}}{(r-1)(b-2)}, \\
& y=\frac{r P+(b-1)\left(A_{i} B_{k 2}\right)+\left(A_{i} B_{k 1}\right)-A_{i}}{(r-1)(b-2)},
\end{aligned}
$$

para $b>2$.

c) Duas subparcelas perdidas que receberam tratamentos $A$ e $B$ diferentes:

$$
\begin{aligned}
& x=\frac{r P_{1}+b\left(A_{i} B_{k_{1}}\right)-A_{i_{1}}}{(r-1)(b-1)}, \\
& y=\frac{r P_{2}+b\left(A_{i} B_{k_{2}}\right)-A_{i_{2}}}{(r-1)(b-1)} .
\end{aligned}
$$


d) Duas subparcelas perdidas que receberam tratamentos A diferentes e mesmo tratamento $B$ :

$$
\begin{aligned}
& x=\frac{r P_{1}+b\left(A_{1} B_{k_{1}}\right)-A_{11}}{(r-1)(b-1)}, \\
& y=\frac{r P_{2}+b\left(A_{1} B_{k_{2}}\right)-A_{12}}{(r-1)(b-1)} .
\end{aligned}
$$

e) Duas subparcelas perdidas que receberam mesmo tratamento $A$ e tratamentos $B$ diferentes:

$$
\begin{aligned}
& x=\frac{(r-1)(b-1)\left[r P_{1}+b\left(A_{i} B_{k 1}\right)-A_{i}\right]-r P_{2}-b\left(A_{i} B_{k 2}\right)+A_{i}}{(r-1)^{2}(b-1)^{2}-1} \\
& y=\frac{(r-1)(b-1)\left[r P_{2}+b\left(A_{i} B_{k 2}\right)-A_{i}\right]-r P_{1}-b\left(A_{1} B_{k 1}\right)+A_{i}}{(r-1)^{2}(b-1)^{2}-1}
\end{aligned}
$$

f) Duas subparcelas perdidas que receberam mesmos tratamentos $A$ e $B$ :

$$
\begin{aligned}
& x=\frac{(r-1) P_{1}+P_{2}+b\left(A_{1} B_{k}\right)-A_{1}}{(b-1)(r-2)} \\
& y=\frac{(r-1) P_{2}+P_{1}+b\left(A_{1} B_{k}\right)-A_{1}}{(b-1)(r-2)}
\end{aligned}
$$


Os termos das fórmulas têm os seguintes significados:

$A_{i}$ : total das observaçöes relativas ao tratamento $A$ atribuído à parcela com a subparcela perdida;

P: total das subparcelas presentes na parcela onde foi perdida uma observação;

$A_{i} B_{k}$ : total das observações relativas aos tratamentos $A_{i}$ e $B_{k}$ cor respondentes à subparcela perdida.

Nos casos de duas subparcelas perdidas $(x$ e $y)$, os termos têmosmesmossignıficados, sendo acompanthados do índice I quan do se referem à subparcela perdida $x$ e do indice 2 quando se referem à subparcela perdida y.

Esta autora verificou ainda que as fórmulas dos itens $\underline{c}$ e d não diferem da obtida para a estimativa de uma ünica subparce la perdida, e que a fórmula apresentada no item a é suficiente para as obtenções das estimativas das subparcelas perdidas, quando $x$ e $y$ receberem tratamentos $A$ e $B$ diferentes, ou tratamentos $A$ diferentes e mesmo tratamento $B$.

FREITAS (1977) e FREITAS et alii (1978), ao analisarem um ensaio em parcelas subdivididas com I tratamentos $T$ aplicados às parcelas, dispostos em $J$ blocos casualizados, $K$ tratamentos T' aplicados às subparcelas, estimaram o valor de uma parcela perdida através da fórmula apresentada por YÁTES (1933), para delineamentos em blocos casualizados, ou seja, 


$$
z=\frac{I T+J B-G}{(I-I)(J-I)},
$$

onde, z: estimativa da parcela perdida;

$T$ : total das parcelas restantes no tratamento $T$;

B: total das parcelas restantes no bloco $\mathrm{J}$;

G: total das parcelas disponíveis.

Estes autores, aplicando o método da soma mínima de quadrados, para o caso de uma parcela perdida constituida de duas subparcelas, determinaram valores das estimativas das subparcelas perdidas.

HADDAD (1978), tornando mínima a soma de quadrados do resfduo (b), deduziu fórmulas para as estimativas das subparcelas perdidas em delineamentos com parcelas subdivididas, as quais compöem uma parcela qualquer, como sendo:

a) Uma parcela perdida constituída de duas ou mais subparcelas:

$$
x+y+\ldots+w=\frac{I T+J B-G}{(I-1)(J-1)} .
$$

Percebe-se que esta fórmula ẻ a mesma utilizada por FREITAS (1977) e FREITAS et alii (1978).

b) Duas subparcelas perdidas, as quałs compõem uma parcela qual quer:

$$
x=\frac{I T+3 B-G+(I-1) P_{1}-(I-I) P_{2}}{2(I-1)(J-1)}
$$




$$
y=\frac{I T+J B-G-(I-I) P_{1}+(I-I) P_{2}}{2(I-I)(J-1)} .
$$

c) Três subparcelas perdidas, as quais compõem uma parcela qual quer:

$$
\begin{aligned}
& x=\frac{2(I-1) P_{1}-(I-I) P_{2}-(I-I) P_{3}+I T+J B-G}{3(I-I)(J-I)} \\
& y=\frac{2(I-1) P_{2}-(I-1) P_{1}-(I-I) P_{3}+I T+J B-G}{3(I-1)(J-1)} \\
& z=\frac{2(I-1) P_{3}-(I-1) P_{1}-(I-1) P_{2}+I T+J B-G}{3(I-I)(J-I)}
\end{aligned}
$$

d) Para mais de três subparcelas perdidas, as quais compōem uma parcela qualquer:

$$
\begin{aligned}
& x=\frac{(I-1)\left[(K-1) P_{1}-P_{2}-\ldots-P_{W}\right]+I T+J B-G}{K(I-1)(J-1)} \\
& y=\frac{(I-1)\left[(K-I) P_{2}-P_{1}-\ldots-P_{W}\right]+I T+J B-G}{K(I-I)(J-I)} \\
& w=\frac{(I-1)\left[(K-1) P_{W}-P_{1}-\ldots-P_{U}\right]+I T+J B-G}{K(I-I)(J-1)}
\end{aligned}
$$

Os termos das förmulas têm os seguintes significados: 


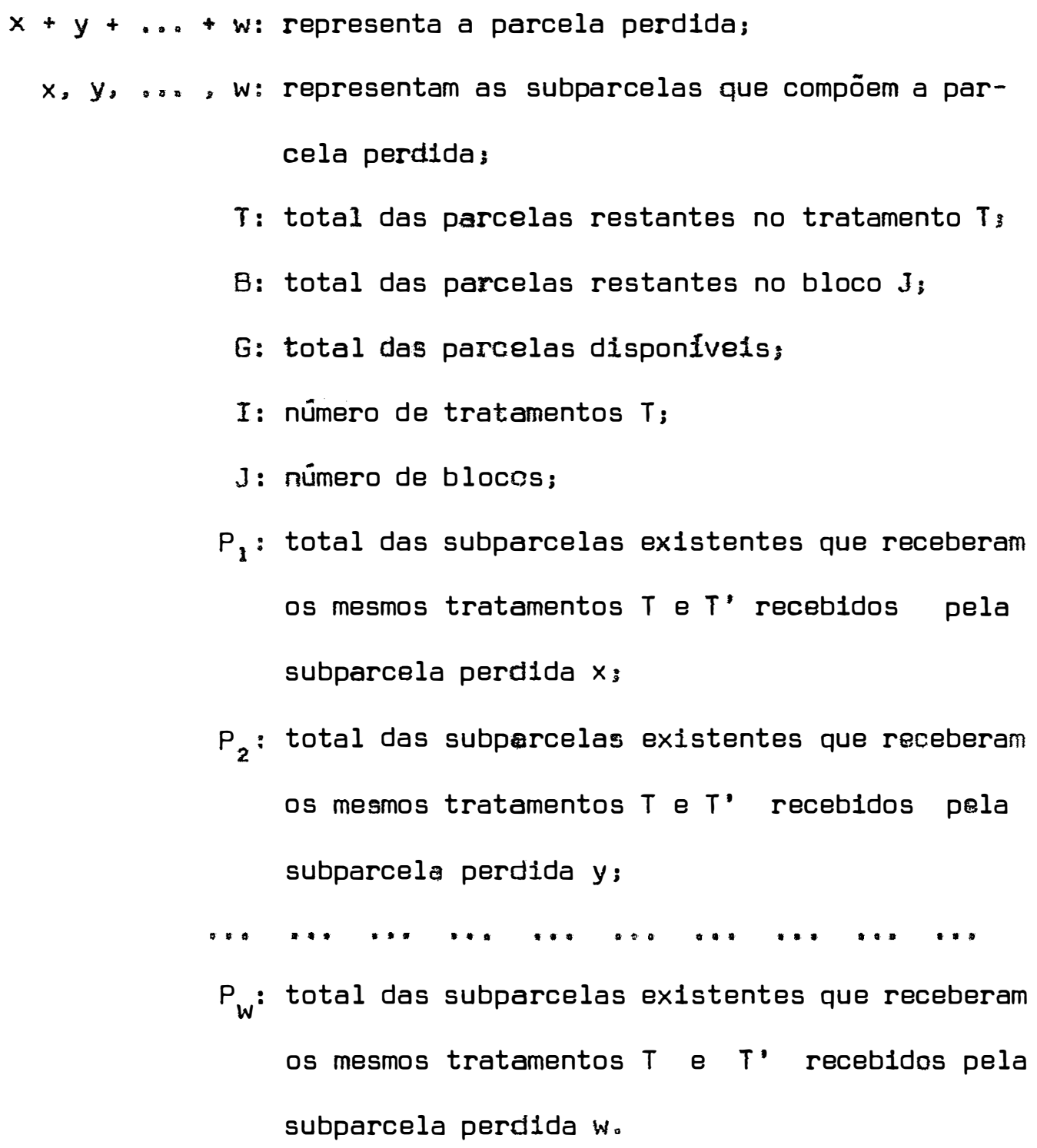

CAMPOS (1964) af1rmou que uma solução, embora um tan to drástica, serla eliminar os blocos (se for o caso) onde figuram as parcelas perdidas, se o número de parcelas componentes dos blocos restantes fosse suficiente para se efetuar a anälise estatísti ca a bom termo. 
Este autor salientou que o mais acertado é analisar o experimento com os dados restantes, levando-se em consideração a perda ocorrida.

YATES (1933), ao analisar um experimento em blocos casualizados com uma parcela perdida, afirmou que se o valor estima do for inserido no lugar da observação perdida, e se os dados forem analisados como se nenhuma observação estivesse ausente, algumas im portantes propriedades se mantēm:

a) as estimativas dos efeitos de tratamentos e efeitos de blocos são as mesmas que as obtidas pelo método correto dos qua drados mínimos;

b) a soma de quadrados do resíduo é exatamente a mesma que a dada pelo procedimento correto;

c) os números exatos de graus de liberdade, associados ao total e ao resíduo, se obtèm subtraindo uma unidade de cada。 respectivamente.

Este autor mostrou que a soma de quadrados de tratamentos, quando se utilizam todos os dados "completos", está sempre superestimada.

ANDERSON (1946) afirmou que, se a estimativa da subparcela perdida for usada no lugar da observação perdida, todas as somas de quadrados, exceto a do resíduo (b), estão superestimadas;e que a estimativa imparcial de qualquer soma de quadrados é determi- 
nada subtraindo-se a tendēncia ("bias"), dada pela expressão:

$$
\left(y-y_{1}\right)^{2} s\left(x_{1}^{2}\right)
$$

onde:

a) Para os tratamentos B dispostos nas subparcelas:

$$
\begin{gathered}
y_{1}=\frac{r \alpha\left(R_{1} A_{1}\right)+\alpha \beta\left(A_{1} B_{1}\right)-\beta B_{1}+G}{(\beta-1)(r \alpha-\alpha+1)}, \\
S\left(x_{1}^{2}\right)=\frac{(\beta-1)(r \alpha-\alpha+1)}{r \alpha \beta} .
\end{gathered}
$$

b) Para a interação $A \times B$ :

$$
\begin{gathered}
y_{1}=\frac{\operatorname{ra}\left(R_{1} A_{1}\right)+B B_{1}-G}{(r \alpha-1)(B-1)} . \\
S\left(x_{1}^{2}\right)=\frac{(\beta-1)(r \alpha-1)}{r \alpha \beta} .
\end{gathered}
$$

sendo, $B_{1}$ : total das observações existentes com o tratamento $b_{1}$;

G: total das observações existentes.

STEEL e TORRIE (1960), ao abordarem um ensaio em par celas subdivididas com subparcelas perdidas, sugeriram que a análise de variância deve ser feita após a introdução das estimativas dos valores das observaçöes perdidas; e um grau de liberdade deve ser 
subtraido do erro (b) para cada subparcela perdida do ensaio.

Estes autores afirmaram que somente a soma de quadra dos do erro (b) é imparcial, sendo que o mesmo não acontece com as demais somas de quadrados.

PINHO (1973), aplicando o método do residuo condicio nal ao experimento analisado por ANDERSON (1946), demonstrou que a soma de quadrados para a interação AxB pode ser determinada correta mente através da fórmula dada por aquele autor, não acontecendo o mesmo com as demais.

Para o caso de uma subparcela perdida, a autora determinou fórmulas para as correções das somas de quadrados através do método do resíduo condicional, como sendo,

a) Correção para a soma de quadrados da interação AxB:

$$
U_{A B}=\frac{(r a-1)(b-1)}{r a b}\left(y_{1}-y_{2}\right)^{2}
$$

b) Correção para a soma de quadrados de tratamentos $B$ dispostos nas subparcelas:

$$
U_{B}=\frac{b-1}{r a b} y_{1}^{2}+2 y_{1} \frac{b B_{k}-G}{\operatorname{rab}}-\frac{(b-1)(r a-1)}{\operatorname{rab}} y_{2}^{2}+\frac{b-1}{b} \cdot y_{3}^{2} \text {. }
$$

onde, $y_{1}, y_{2}, y_{3}$ : são estimativas das observações perdidas, obtidas através do método do resíduo condicional. 
A autora concluiu que as somas de quadrados sem correção, exceto a soma de quadrados da interação $A \times B$, não estão sempre superestimadas como afirmou ANDERSON (1946): podem estar super ou subestimadas.

A mesma autora disse que, quando se tem mais do que uma subparcela perdida, não é conveniente determinar förmulas para correçōes das somas de quadrados, sendo mais simples determinar as somas de quadrados diretamente atravës de Algebra de Matrizes.

HADDAD (1978) demonstrou que a soma de quadrados de tratamentos T' ajustada é igual à soma de quadrados de tratamentos $T^{\prime}$, quando determinada ignorando-se a parcela perdida; e que a soma de quadrados da interação TXT' ajustada é facilmente obtida pela se guinte expressão:

$$
\begin{aligned}
\operatorname{SQT} T T^{\prime}\left(a_{j}\right)=\operatorname{SQTotal}(\text { usual) } & -\operatorname{SQR}(b)-\operatorname{SQT}^{\prime}(\text { usual) - } \\
& -\operatorname{SQP}_{(i, j)} \text { (usual) }
\end{aligned}
$$

onde, SQTXT'(aj): soma de quadrados da interação TXT' ajustada;

SQTotal(usual): soma de quadrados de totais:

SQR(b): soma de quadrados do residuo $\{b\}$;

SQT'(usual): soma de quadrados de tratamentos T' dispostos nas subparcelas;

$\operatorname{SQP}_{(i, j)}$ (usual): soma de quadrados de parcelas que receberam o $i$ ésimo nível do tratamento $T$ no j-ésimo bloco,

sendo que a notação (usual) indica que a soma de quadrados foi determinada ignorando-se a parcela perdida. 
A mesma autora afirmou que, para cada parcela perdida, o resíduo (a) perde um grau de liberdade e o residuo (b) perde tantos graus de liberdade quantas forem as subparcelas que compöem a parcela perdida.

COCHRAN e COX (1976) adotaram o seguinte procedimento para analisar um experimento com uma observação perdida:

Inicialmente "estimar" o valor da observação perdida através de förmula.

Essa estimativa é colocada no lugar da observação per dida, e a análise de varianncia é efetuada de maneira usual, exceto pela mudança do nümero de graus de liberdade do erro residual.

A soma de quadrados deve ser corrigida através de mé todos especiais, possibilitando a aplicação correta do teste $F$ e do teste $\underline{t}$.

PIMENTEL GOMES (1978), ao analisar um ensaio em parcelas subdivididas, com observação perdida, adotou o procedimento apresentado por COCHRAN e COX (1976), e afirmou que somente o quadrado médio do resíduo (b) fica corretamente estimado, mas todos os outros estão superestimados, sendo o erro cometido geralmente desprezível, podendo-se usar o mëtodo do residuo condicional para se obterem os valores exatos.

Quando a comparação entre médias de tratamentos, dis postas nas parcelas, é aquela em que mais se tem interesse, pode-se com maior rigor, segundo PIMENTEL GDMES (1978), considerar perdida 
toda a parcela onde se perdeu uma subparcela e calcular o valor a ser utilizado na análise, pela fórmula:

$$
y=\frac{r B+n T-G}{(r-1)(n-1)}
$$

onde, y: estimativa da parcela perdida;

r: número de repetições;

B: total das parcelas restantes no bloco em que figura a parcela perdida:

$\mathrm{n}$ : número a de tratamentos $\mathrm{A}$;

$T$ : total do tratamento de parcela perdida nos outros blocos;

G: total das parcelas disponíveis.

Para experimentos em blocos casualizados com parcelas subdivididas, ANDERSON (1946) apresentou fórmulas para estimati vas de variâncias das estimativas de contrastes entre duas médias de tratamentos, em que um deles perdeu uma observação, nos casos de:

a) Duas médias dos tratamentos $A$ dispostos nas parcelas:

$$
\nabla\left(\hat{a}_{i}-\hat{a}_{i},\right)=\frac{2}{\Gamma B}\left[E_{a}+\frac{E_{b}}{2(r-1)(B-1)}\right] ;
$$

b) Duas médias dos tratamentos B dispostos nas subparcelas:

$$
\nabla\left(\sigma_{j}-\sigma_{j}\right)=\frac{2 E_{b}}{r \alpha}\left[1+\frac{\beta}{2 \alpha(r-1)(\beta-1)}\right] ;
$$


c) Duas médias de tratamentos $B$ no mesmo nível de $A$ :

$$
\nabla\left(\hat{a_{i} b_{j}}-{\widehat{a_{1} b_{j}}}=\frac{2 E_{b}}{r}\left[I+\frac{\beta}{2(r-1)(B-1)}\right]\right.
$$

d) Duas médias de tratamentos A no mesmo nível de B:

$$
\hat{O}\left(\hat{a}_{i} b_{j}-\hat{a}_{i}, b_{j}\right)=\frac{2 E_{a}}{r \beta}+\frac{2 E_{b}}{r \beta}\left[(\beta-1)+\frac{\beta^{2}}{2(r-1)(\beta-1)}\right]
$$

onde, $E_{a}$ : quadrado médio do resíduo $(a)$;

$E_{b}$ : quadrado médio do resíduo (b).

COCHRAN e COX (1976) apresentaram förmulas idênticas às apresentadas por ANDERSON (1946) para estimativas de vartâncias das estimativas de contrastes entre duas médias de tratamentos, em que um deles perdeu uma observação, exceto a do item $\underline{c}$, que é dada por:

$$
\nabla\left(\hat{a_{i} b_{j}}-\hat{a}_{i} b_{k}\right)=\frac{2 E_{b}}{r}\left[1+\frac{B}{2 a(r-1)(b-1)}\right] .
$$

PINHO (1973) deduziu förmulas para estimativas de va riāncias das estimativas de contrastes entre duas médias de tratamen. tos, em que um deles perdeu uma observação, nos casos de:

a) Duas médias dos tratamentos $T$ dispostos nas parcelas:

$$
\nabla\left(E_{i}-E_{i},\right)=\frac{2}{J K}\left[E_{a}+\frac{(I-2) E_{b}}{2 I(J-I)(K-I)}\right] \text {; }
$$


b) Duas médias dos tratamentos $T^{\prime}$ dispostos nas subparcelas:

$$
V\left(E_{k}^{\prime}-E_{k}^{\prime}\right)=\frac{2 E_{b}}{I J}\left[I+\frac{K}{2 I(J-I)(K-I)}\right] ;
$$

c) Duas médias de tratamentos $T$ ' no mesmo nível de tratamento $T:$

$$
\nabla\left[\left(E_{k}^{\prime}-E_{k}^{\prime}\right) / E_{i}\right]=\frac{2 E_{b}}{J}\left[1+\frac{k}{2(J-1)(k-1)}\right] ;
$$

d) Duas médias de tratamentos $T$ no mesmo nível de tratamento $T^{\prime}:$

$$
\nabla\left[\left(E_{1}-E_{i},\right) / E_{k}^{\prime}\right]=\frac{2 E_{a}}{J K}+\frac{2 E_{b}}{J K}\left[(K-1)+\frac{I K^{2}-2}{2 I(J-1)(K-1)}\right] ;
$$

onde, $E_{a}$ : quadrado médio do resíduo $(a)$; $E_{b}$ : quadrado médio do resíduo (b).

A autora verificou que as förmulas dos itens a e d não são concordantes com as apresentadas por ANDERSON (1946) e COCH RAN e COX (1976); e que a do Item c é concordante com a apresentada por ANOERSON (1946) e discordante da estabelecida por COCHRAN e COX (1976).

$$
\text { HADDAD (1978) deduziu fórmulas para estimativas das }
$$
variânciæ̇ das estimativas de contrastes entre duas médias de tratamentos, em que um deles perdeu uma observação, em experimento em blo 
cos casualizados com parcelas subdivididas, nos casos de:

a) Duas médias dos tratamentos $T$ dispostos nas parcelas:

$$
\nabla\left(E_{1}-E_{I^{\prime}}\right)=\frac{2}{J K}\left[1+\frac{I}{2(I-1)(J-1)}\right] \text { QMR(a). }
$$

Nota-se que esta fórmula é idêntica àquela para se calcular as estimativas das variâncias das estimativas de contrastes entre médias de tratamentos, em experimentos em blocos casualizados com uma parcela perdida, apresentada por YATES (1933).

b) Duas médias dos tratamentos T' no mesmo nível do tratamento T:

$$
\nabla\left[\left(E_{k}^{\prime}-E_{k}^{\prime}\right) / E_{1}\right]=\frac{2}{j-1} \operatorname{QMR}(b) .
$$

onde, QMR(a): quadrado médio do resíduo (a): QMR(b): quadrado médio do resíduo $(b)$. 
22.

4. MATERIAIS E METODOS

\section{1 - Materiais}

Para comprovação dos resultados foram utilizados dados de dois ensaios em delineamento inteiramente casualizado com par celas subdivididas, os quais constam no Apêndice (Tabelas 12 e 13). O experimento A foi compilado de PIMENTEL GOMES(1978), e nele foi aplicada toda a metodologia estudada para os seguintes casos:

a) Uma subparcela perdida;

b) Duas subparcelas perdidas numa mesma parcela composta de $k>2$ subparcelas:

c) Duas subparcelas perdidas que receberam tratamentos $T$ e $T$ ' diferentes; 
d) Duas subparcelas perdidas que receberam tratamentos T diferentes e mesmo tratamento $T^{\prime}$ '

e) Duas subparcelas perdidas que receberam mesmo tratamento $T$ e tratamentos $T^{\prime}$ diferentes, em parcelas distintas;

f) Duas subparcelas perdidas que receberam mesmos tratamentos $T \& T^{\prime}:$

g) Uma parcela perdida composta de $k>2$ subparcelas.

O experimento B foi cedido pela Superintendência Geral do PLANALSUCAR em Piracicaba (SP), tendo sido aplicada a metodologia estudada para o caso de uma parcela perdida constituída de duas subparcelas.

\section{2 - Métodos}

Seja um experimento em delineamento inteiramente casualizado com parcelas subdivididas, tendo-se I tratamentos $T$ aplicados às parcelas dispostos em $J$ repetiçöes, $K$ tratamentos $T$ ' aplicados às subparcelas.

Cada subparcela é representada pelo modelo matemático:

$$
\begin{gathered}
x_{i j k}=m+t_{i}+e_{i j}+t_{k}^{\prime}+t t_{(i k)}^{\prime}+e_{i j k} \quad(4.2 . a) \\
i=1,2, \ldots, I, j=1,2, \ldots, j ; \\
k=1,2, \ldots, k:
\end{gathered}
$$


onde, $x_{i j k}$ : valor observado da ik-ésima subparcela na j-ésima repetição:

m: média geral teórica;

$t_{i}$ : efeito do i-ésimo tratamento $T:$

$e_{i j}$ : efeito do i-ésimo tratamento $T$ na j-ésima repetição, on de se supõe $e_{i j}$ com distribuição normal de média zero e variância $\sigma_{a}^{2}$, ou seja, $e_{i j} \cap N\left(0, \sigma_{a}^{2}\right)$;

tt' (ik): efeito da interação do i-ésimo tratamento $T$ com o k-ési mo tratamento $T^{\prime}$;

$e_{i j k}$ : efeito da ik-ésima subparcela na j-ésima repetição, onde se supõe $e_{i j k}$ com distribuição normal de média zero e variância $\sigma_{b}^{2}$, ou seja, $e_{i j k} \cap N\left(0, \sigma_{b}^{2}\right)$.

\subsection{1 - Equações normais}

Foram obtidas através do método da soma mínima de qua drados, considerando-se as seguintes restrições:

$$
\begin{aligned}
& \sum_{i} E_{i}=0 ; \quad \sum_{k} E_{k}^{\prime}=0 \\
& \sum_{i, k} t t_{(i k)}^{\prime}=0 \quad \sum_{i} t t_{(i k)}^{\prime}=0 \\
& \sum_{k} t t_{(i k)}^{\prime}=0 ; \sum_{i, j} \vec{e}_{i j}=0 \\
& \sum_{i} \hat{e}_{i j}=0 ; \quad \sum_{j} \hat{e}_{i j}=0 \text {. }
\end{aligned}
$$


As equações normais obtidas foram as que se seguem:
a)
b)

$$
J K \tilde{m}+J K E_{1}=T_{1}
$$
c)
$K \bar{m}+K E_{1}+K \hat{e}_{i j}=P_{i j}$
d)
$I J \hat{m}+I J E_{k}^{\prime}=T_{k}^{\prime}$
e) $J \tilde{m}+J t_{1}+J t_{k}^{\prime}+J t t_{(I k)}^{\prime}=T T_{(1 k)}$.

onde, G: total geral:

$T_{1}$ : total do i-ésimo tratamento $T$;

$P_{i j}$ : total das parcelas que receberam o i-ésimo tratamento $T$ na j-ësima repetição;

$T_{k}^{\prime}$ : total do k-ésimo tratamento $T^{\prime}$ :

$T^{\prime}(i k)$ : total das subparcelas relatıvas ao $i$-ésimo tratamento $T$ com o k-ésimo tratamento $T^{\prime}$.

\subsection{2 - Estimativas dos efeitos dos parâmetros}

Partindo-se das equações normais, obtiveram-se as es timativas dos efeitos dos parâmetros, segundo o modelo matemático proposto, ou sejam,

$$
\hat{m}=\frac{G}{I J K}
$$


26.

$$
\begin{aligned}
t_{i} & =\frac{T_{i}}{J k}-\hat{m} \\
\hat{e}_{i j} & =\frac{P_{i j}}{k}-E_{i}-\hat{m}, \\
E_{k}^{\prime} & =\frac{T_{k}^{\prime}}{I J}-\hat{m} \\
t t_{(i k)}^{\prime} & =\frac{T T_{(i k)}^{\prime}}{J}-E_{i}-E_{k}^{\prime}-\hat{m}
\end{aligned}
$$

\subsection{3 - Estimativas das subparcelas perdidas}

Utilizando-se o método da soma mínima de quadrados,

ou seja, minimizando-se a soma de quadrados do residuo (b) [SQR(b)]. foram determinadas as fórmulas para as estimativas das subparcelas perdidas .

Para melhor compreensão da metodologia proposta, foi estudado, como a seguir, o caso de duas subparcelas perdidas em tra tamentos $T$ e T' diferentes.

Seja o mesmo experimento descrito em 4.2, representa do pelo modelo em $\{4,2 . a\}$.

Tornando minima a SQR (b), foram obtidas as estimati vas $\left(x_{1}, x_{2}\right)$ para as subparcelas perdidas, através das fórmulas dadas por:

$$
x_{1}=\frac{J P_{1}+K\left(T_{1} T_{k 1}^{0}\right)-T_{1}}{(3-1)(K-1)}
$$




$$
x_{2}=\frac{J P_{2}+K\left(T_{i} T_{k_{2}}^{\prime}\right)-T_{i}}{(J-1)(K-1)}
$$

Eliminando-se do modelo, dado pela expressão (4.2.a) o parâmetro $t t_{(i k)}$ e aplicando-se o método da soma mínima de quadra dos, obtiveram-se novas estimativas $\left(y_{1}, y_{2}\right)$ para as subparcelas perdidas, dadas por:

$$
\begin{aligned}
& y_{1}=\frac{(I J-I)(K-I)\left(I J P_{1}+K T_{K_{1}}^{\prime}-G\right)-I J P_{2}-K T_{K_{2}}^{\prime}+G}{(I J-I)^{2}(K-I)^{2}-1} \quad(4 \cdot 2.3 . c) \\
& y_{2}=\frac{(I J-I)(K-I)\left(I J P_{2}+K T_{K_{2}}^{\prime}-G\right)-I J P_{1}-K T_{K_{1}}^{\prime}+G}{(I J-I)^{2}(K-I)^{2}-1} \quad \text { (4.2.3.d) }
\end{aligned}
$$

Considerando-se o modelo em $(4.2 . a)$, sem os parâmetros $t_{k}^{\prime}$ e $t t_{(i k)}^{\prime}$ e aplicando-se o método da soma mínima de quadrados, obtiveram-se novas estimativas $\left(z_{1}, z_{2}\right)$ para as subparcelas perdidas, dadas por:

$$
\begin{aligned}
& z_{1}=\frac{P_{1}}{k-1} \\
& z_{2}=\frac{P_{2}}{k-1}
\end{aligned}
$$

onde, $P_{1}$ : total das subparcelas existentes na parcela onde foi perdida a subparcela $x_{1}$;

$P_{2}$ : total das subparcelas existentes na parcela onde foi perdida a subparcela $x_{2}$; 


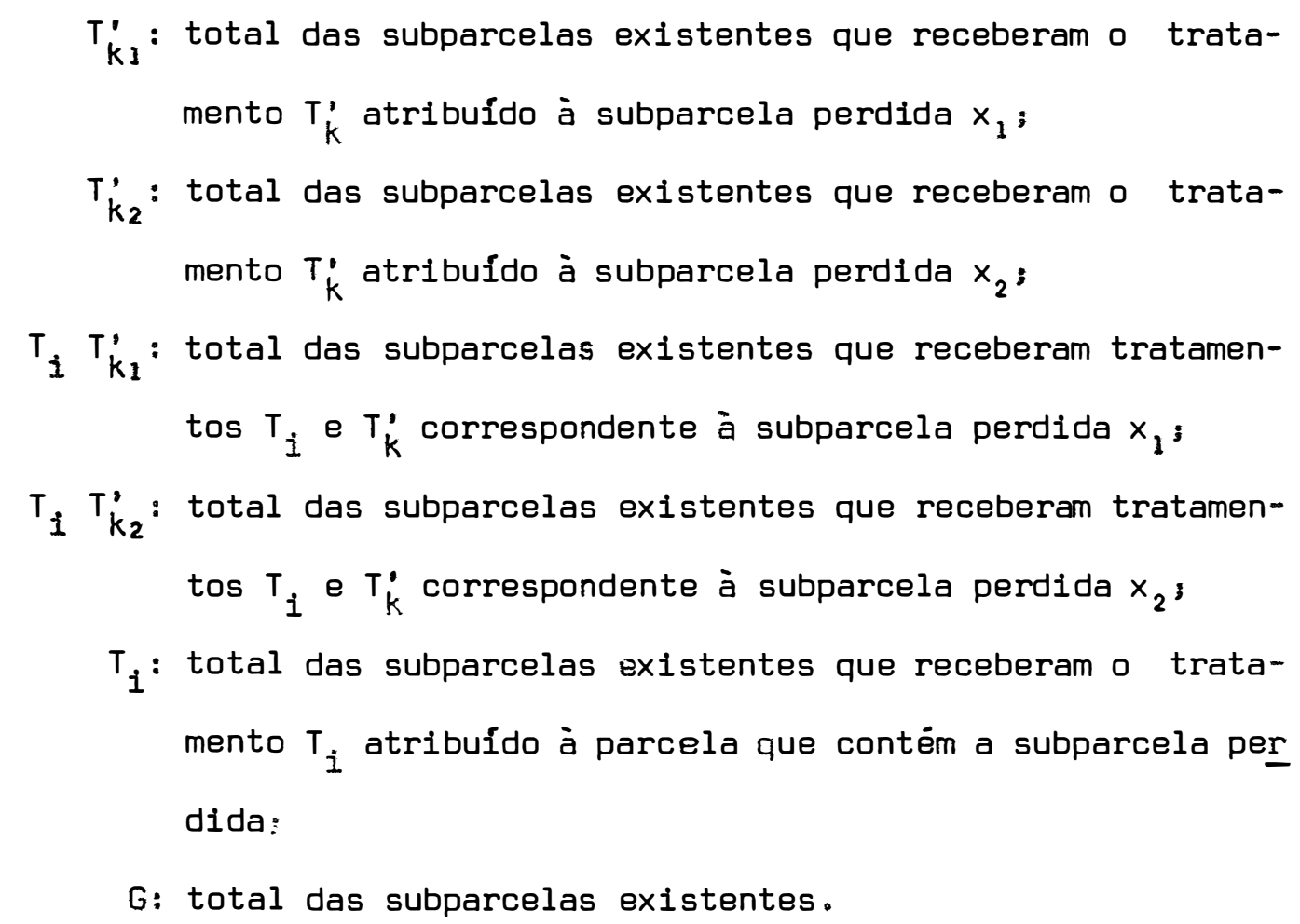

\subsection{4 - Obtenção das somas de quadrados ajustadas}

As somas de quadrados ajustadas foram obtidas através do método do resíduo condicional de R.A. Fisher, que è baseado no método da soma mínima de quadrados.

Em seguida são apresentadas as notações para somas de quadrados a serem utilizadas no desenvolvimento deste trabalho:

$$
\begin{aligned}
\operatorname{SQT} \text { Tatamento (usual) } & =\operatorname{SQT}(\text { usual) } \\
\operatorname{SQParcelas}_{(i, j)} \text { iusual) } & =\operatorname{SQP}(\text { usual) } \\
\text { SQResíduola } & =\operatorname{SQR}(a)
\end{aligned}
$$




$$
\begin{aligned}
& \text { SQT ratamentos } T^{\prime}(x)=\operatorname{SQT} T^{\prime}(x) \\
& \text { SQTratamentos } T(x)=\operatorname{SQT}(x)
\end{aligned}
$$

SQTratamentos $T^{\prime}(x)$ e Tratamentos $T^{\prime}(x)=\operatorname{SQT}, T^{\prime}(x)$

$$
\begin{aligned}
\text { SQInteração } \operatorname{TxT}^{\prime}(x) & =\operatorname{SQT\times T} \cdot(x) \\
\text { SQTratamentos } T^{\prime}(\text { usual) } & =\operatorname{SQT} T^{\prime} \text { (usual) } \\
\operatorname{SQParcelas}_{(i, j)}(x) & =\operatorname{SQP}(x) \\
\operatorname{SQRes}_{\text {Iduo }}(b) & =\operatorname{SQR}(b)
\end{aligned}
$$

onde, $x=x_{1}, x_{2}, \ldots, x_{k}$

0 indice $(x)$, indica que as somas de quadrados foram calculadas levando-se em conta as estimativas das subparcelas perdi das, enquanto que, o Indice (usual) indica que foram calculadas somente com valores das subparcelas disponíveis.

Para a correção da soma de quadrados de tratamento $T$, considerou-se um experimento em delineamento inteiramente casuali zado com $k$ amostras por parcela, cujo modelo matemático é dado por:

$$
x_{i j k}=m+t_{i}+e_{i j k}
$$

Isto se justifica, uma vez que a soma de quadrados de tratamentos $T$ pertence à primeira parte da análise de variância de 
um delineamento inteiramente casualizado com parcelas subdivididas: e a perda de uma ou mais amostras neste tipo de delineamento em nada afetará a soma de quadrados de tratamentos, isto é, sempre se ob tém uma soma de quadrados ajustada.

Determinou-se, portanto, a soma de quadrados de resí duo (a), dada por:

$$
\operatorname{SQR}(a)=\operatorname{SQP}(\text { usual) - SQT(usual). }
$$

Partindo-se do modelo, dado pela expressão (4.2.a), e levando-se em consideração as estimativas das subparcelas perdidas, determinou-se a soma de quadrados do resíduo (b),

$$
\operatorname{SQR}(b)=\sum_{i j k} x_{i j k}^{2}-\operatorname{SQP}\left(\hat{m}, \hat{t}_{i}, \hat{e}_{i j}, \hat{t}_{k}^{\prime}, t t_{i k}^{\prime}\right)
$$

ou seja,

$$
\begin{aligned}
& \operatorname{SQR}(b)=\operatorname{SQR}_{1}=\operatorname{SQTotal}(x)-\operatorname{SQP}(x)-\operatorname{SQT}^{\prime}(x)-\operatorname{SQT} T^{\prime}(x) 。 \\
& \text { Como se perdem tantos graus de liberdade para o resi }
\end{aligned}
$$

duo (b) quantas forem as subparcelas perdidas, o quadrado médio do re siduo (b) fica sempre corretamente estimado, mas os outros, segundo PINHO (1973), estão super ou subestimados。

Para corrigir a $\operatorname{SQTX}^{\prime}(x)$, reduziu-se o modelo, dado pela expressão $(4.2 . a)$, para

$$
x_{i j k}=m+t_{i}+e_{i j}+t_{k}^{\prime}+e_{i j k}
$$


Utilizando-se o método descrito em 4.2.3, determinaram-se novas estimativas (y) para as subparcelas perdidas, com as quais se obteve nova soma de quadrados para resíduo, denominada de $\mathrm{SQR}_{2}$, dada por:

$$
\operatorname{SQR}_{2}=\sum_{i j k} x_{i j k}^{2}-\operatorname{SQP}\left(\tilde{m}, t_{i}, \hat{e}_{i j}, t_{k}^{\prime}\right),
$$

ou seja,

$$
\operatorname{SQR}_{2}=\operatorname{SQTotal}(y)-\operatorname{SQP}(y)-\operatorname{SQT}(y) \text {, }
$$

onde, $y=y_{1}, y_{2}, \ldots, y_{k}$.

0 indice $(y)$ indica que as somas de quadrados foram determinadas levando-se em consideração as estimativas (y) das subparcelas perdidas.

Logo, a soma de quadrados da interação TxT' ajustada,

ë dada por:

$$
S Q T \times T^{\prime}(a j)=S Q R_{2}-S Q R_{1}
$$

0 indice ( $a j$ ) indica que as somas de quadrados foram ajustadas.

Para corrigir a SQT' $(x)$, reduziu-se o modelo(4.2.4.b) para:

$$
x_{i j k}=m+t_{i}+e_{i j}+e_{i j k}
$$

Empregando-se o método descrito em 4.2.3, determinaram-se novas estimativas (z) para as subparcelas perdidas, com as 
quais se obteve nova soma de quadrados para resíduo, denominada de $\mathrm{SQR}_{3}$, dada por:

$$
\operatorname{SQR}_{3}=\sum_{i j k} x_{i j k}^{2}-\operatorname{SQP}\left(\hat{m}, E_{i}, \hat{e}_{i j}\right)
$$

ou seja,

$$
\operatorname{SQR}_{3}=\operatorname{SQTotal}(z)-\operatorname{SQP}(z),
$$

onde, $z=z_{1}, z_{2}, \ldots, z_{k}$.

0 indice $(z)$ indica que as somas de quadrados foram determinadas levando-se em consideração as estimativas $(z)$ das subparcelas perdidas.

Portanto, a soma de quadrados de tratamentos T' ajus tada, é dada por:

$$
\operatorname{SQT} \cdot(a j)=S Q R_{3}-S Q R_{2} .
$$

Quando foram perdidas k subparcelas, as quais consti tuiam uma parcela qualquer, a soma de quadrados de tratamentos T'ajustada, foi obtida de maneira usual, segundo HADDAD (1978).

Para os casos onde foram perdidas uma ou duas subpar celas em parcelas de $k>2$ subparcelas, a soma de quadrados de tratamentos T' ajustada foi obtida pela seguinte expressão:

$$
\operatorname{SQT}^{\prime}(a j)=\operatorname{SQTotal}\left(\text { usual) }-\operatorname{SQP}\left(\text { usual) }-\operatorname{SQTXT}^{\prime}(\mathrm{aj})-\mathrm{SQR}_{\mathbf{l}}\right. \text {. }\right.
$$

A demonstração será efetuada a seguir, considerando-se que foram perdidas duas subparcelas que receberam tratamentos $T$ 
e T' diferentes.

$$
\begin{aligned}
& \text { Substituindo-se os valores de } \mathrm{SQR}_{3} \text { e } \mathrm{SQR}_{2} \text {, obteve-se: } \\
& \operatorname{SQT} \cdot(a j)=\operatorname{SQTotal}\left(z_{1}, z_{2}\right)-\operatorname{SQP}\left(z_{1}, z_{2}\right)-\operatorname{SQT\times T}(\operatorname{aj})-\operatorname{SQR} R_{1} . \\
& \text { Desenvolvendo-se as expressöes } \operatorname{SQTotal}\left(z_{1}, z_{2}\right) \text { e } \operatorname{SQP}\left(z_{1}, z_{2}\right)
\end{aligned}
$$

obteve-se,

$$
\begin{aligned}
S Q T \cdot(a j)=z_{1}^{2}+z_{2}^{2} & +\sum_{i j k}^{\prime} x_{i j k}^{2}-\frac{\left(P_{1}+z_{1}\right)^{2}}{k}-\frac{\left(P_{2}+z_{2}\right)^{2}}{k}- \\
& -\frac{1}{k} \sum_{i, j}^{\sum^{\prime}} P_{(1 j)}^{2}-\operatorname{SQTXT}^{\prime}(a j)-S Q R_{1} .
\end{aligned}
$$

Substituindo-se $z_{1}$ e $z_{2}$ pelos seus respectivos valores dados em $(4.2 .3 . e)$ e $(4.2 .3 . f)$, abteve-se,

$$
\begin{aligned}
& \operatorname{SQT}^{\prime}(a j)=\sum_{i j k}^{\prime} x_{i j k}^{2}-\frac{1}{k-1}\left(P_{1}^{2}+P_{2}^{2}\right)-\frac{1}{K} \sum_{i j}^{\prime} P_{(i j)}^{2}- \\
& -\operatorname{SQT} \times T^{\prime}(a j)-\mathrm{SQR}_{1}
\end{aligned}
$$

$$
\text { Somando-se e subtraindo-se uma correção } C=\frac{G^{2}}{I J K-2} \text {, }
$$

ou seja,

$$
\begin{aligned}
\operatorname{SOT} T^{\prime}(a j)=\sum_{i j k}^{\sum^{\prime}} x_{i j k}^{2} & -C-\left[\frac{1}{K-1}\left(P_{1}^{2}+P_{2}^{2}\right)+\frac{1}{K} \sum_{i j} P_{(i j)}^{2}-C\right]- \\
& -\operatorname{SQTXT}^{\prime}(a j)-S Q R_{1},
\end{aligned}
$$

logo, 


$$
\begin{aligned}
\operatorname{SQT}^{\prime}(a j)=\operatorname{SQTotal}(\text { usual) } & -\operatorname{SQP}\left(\text { usual) }-\operatorname{SQT} \times T^{\prime}(a j)-\right. \\
& -\mathrm{SQR}_{1}
\end{aligned}
$$

onde, $\underset{i j k}{\Sigma^{\prime}} x_{i j k}^{2}:$ somatório dos quadrados das subparcelas existentes: $\sum_{i j}^{\prime} P_{(i j)}^{2}$ : somatörio dos quadrados das parcelas onde não ocor reu subparcela perdida.

\subsection{5 - Cālculo das correçōes "U" para a soma de quadrados da interação TxT'}

As correçöes "U" foram obtidas pelo método do resíduo oondicional de R.A. Fisher.

Segundo ANOERSON (1946), PINHO (1973), HADDAD (1978)

e PIMENTEL GOMES (1978), a $\operatorname{SQTXT}^{\prime}(x)$ está sempre superestimada. Logo,

$$
\operatorname{SQTXT}(x)>\operatorname{SQT} \times T^{\prime}(a j)
$$

Portanto, existe um valor "U" tal que,

$$
U=\operatorname{SQT} \times T^{\prime}(x)-\operatorname{SQT} \times T^{\prime}(a j)
$$

Considerando-se o caso onde ocorreu uma subparcela perdida, e substituindo-se a SQTXT' $\left(a_{j}\right\}$ pelo seu valor, obteve-se,

$$
U=\operatorname{SQTXT}(x)-S Q R_{2}+\operatorname{SQR}_{1}
$$

Desenvolvendo-se as expressōes de $\mathrm{SQR}_{2}$ e $\mathrm{SQR}_{1}$, e subs tituindo-se em $(4.2 .5 .6)$, obteve-se, 


$$
U=\frac{(I J-I)(K-1)}{I J K}\left(x_{1}-y_{1}\right)^{2}
$$

Com procedimento análogo, foram obtidas as correções "U" para soma de quadrados da interação TXT', em cada caso estudado neste trabalho.

\subsection{6 - Anālise de variância}

Uma vez determinadas as estimativas das subparcelas perdidas, procederam-se às análises de variância de maneira usual, como se não houvessem subparcelas perdidas.

A seguir foram calculadas as somas de quadrados ajus tadas pela metodologia descrita em 4.2 .4 ou em 4.2.5, para aplicação correta do teste $F$.

Os esquemas das análises de variâncias encontram-se

nas Tabelas 1,2 e 3.

Tabela 1 - Esquema da análise de variância, para o caso em que foi perdida uma subparcela.

\begin{tabular}{|c|c|c|c|}
\hline Fonte de Variação & G.L. & $S \cdot Q$ & $S \cdot Q \cdot(c)$ \\
\hline $\begin{array}{l}\text { Tratamento T } \\
\text { Resíduo (a) }\end{array}$ & $I(J-1)$ & $\begin{array}{l}\text { SQT (usual) } \\
\text { SQR (a) }\end{array}$ & $\begin{array}{l}\text { SQT (usual) } \\
\text { SQR(a) }\end{array}$ \\
\hline $\begin{array}{l}\text { Parcelas } \\
\text { Tratamentos 'T' } \\
\text { Interação TXT' } \\
\text { Resíduo (b) }\end{array}$ & $\begin{array}{c}I J-1 \\
K-1 \\
(I-1)(K-1) \\
I(J-1)(K-1)-1\end{array}$ & $\begin{array}{l}\operatorname{SQP}(\text { usual) } \\
\operatorname{SQT}\left(x_{1}\right) \\
\operatorname{SQTxT^{\prime }}\left(x_{1}\right) \\
\operatorname{SQR}(b)\end{array}$ & $\begin{array}{l}\text { SQP(usual) } \\
\text { SQTxT'(aj) } \\
\text { SQR(b) }\end{array}$ \\
\hline$T \circ t$ a 1 & IJK -2 & & SQTotal (usual) \\
\hline
\end{tabular}


Vê-se que na Tabela I não foi incluída a SQTotal(x), pois ela näo corresponde à soma das diversas fontes de variação.

Os números exatos de graus de liberdade das diversas fontes de variação foram calculados segundo ANDERSON (1946), STEEL e TORRIE (1960) e COCHRAN e COX (1976).

Tabela 2 - Esquema da análise de variância, para os casos em que fo ram perdidas duas subparcelas numa mesma parcela constituída de $k>2$ subparcelas, ou cada uma em parcelas distintas.

\begin{tabular}{|c|c|c|c|}
\hline Fonte de Variação & G.L. & S.Q. & $S \cdot Q \cdot(c)$ \\
\hline $\begin{array}{l}\text { Tratamentos T } \\
\text { ResIduo (a) }\end{array}$ & $\begin{array}{c}I-I \\
I(J-I)\end{array}$ & $\begin{array}{l}\text { SQT (usual) } \\
\text { SQR (a) }\end{array}$ & $\begin{array}{l}\text { SQT (usual) } \\
\text { SQR(a) }\end{array}$ \\
\hline Parcelas & $I J-1$ & SQP (usual) & SQP (usual) \\
\hline $\begin{array}{l}\text { Tratamentos } T^{\prime} \\
\text { Interação } T \times T^{\prime} \\
\text { ResIduo (b) }\end{array}$ & $\begin{array}{c}K-1 \\
(I-I)(K-I) \\
I(J-I)(K-I)-2\end{array}$ & $\begin{array}{l}\operatorname{SQT}(x) \\
\operatorname{SQT} x T^{\prime}(x) \\
\operatorname{SQR}(b)\end{array}$ & $\begin{array}{l}\operatorname{SQT}(a j) \\
\operatorname{SQT} \times T \cdot(a j) \\
\operatorname{SQR}(b)\end{array}$ \\
\hline$T \circ t a 1$ & IJK -3 & & SQTotal (usual) \\
\hline
\end{tabular}

Pelo mesmo motivo descrito para a Tabela 1, deixou- se de incluir a SQTotal $(x)$.

Os números exatos de graus de liberdade das diversas fontes de variação, foram calculados segundo ANDERSON (1946), STEEL e TORRIE (1960) e COCHRAN e COX (1976). 
Tabela 3 - Esquema da análise de variáncla, para o caso em que foi perdida uma parcela composta de $k \geq 2$ subparcelas.

\begin{tabular}{|c|c|c|c|}
\hline Fonte de Variação & G.L. & S.Q. & $S \cdot Q \cdot(c)$ \\
\hline $\begin{array}{l}\text { Tratamentos } T \\
\text { ResIduo (a) }\end{array}$ & $\begin{array}{c}I-1 \\
I(J-I)-1\end{array}$ & $\begin{array}{l}\text { SQT (usual) } \\
\text { SQR(a) }\end{array}$ & $\begin{array}{l}\text { SQT (usual) } \\
\text { SQR(a) }\end{array}$ \\
\hline Parcelas & $I J-2$ & SQP (usual) & SQP (usual) \\
\hline $\begin{array}{l}\text { Tratamentos } T^{\prime} \\
\text { Interação } T \times T^{\prime} \\
\text { Residuo (b) }\end{array}$ & $\begin{array}{c}K-1 \\
(I-I)(K-1) \\
I(J-I)(K-1)-K\end{array}$ & $\begin{array}{l}\operatorname{SQT}(x) \\
\operatorname{SQT} x T^{\prime}(x) \\
\operatorname{SQR}(b)\end{array}$ & $\begin{array}{l}\text { SQT' (usual) } \\
\text { SQT } \times T^{\prime}(a j) \\
\text { SQR (b) }\end{array}$ \\
\hline
\end{tabular}

$T$ o $t$ a 1

SQTotal (usual)

S.Q.(c): tem o mesmo significado estabelecido na Tabela 1.

Nota-se que na Tabela 3 não fol incluido o número de graus de liberdade total, pois ele não corresponde à soma das diver sas fontes de variação.

Pelo mesmo motivo descrito para a Tabela 1, deixou-se de incluir a SQTotal $(x)$.

Os nümeros exatos de graus de liberdade das diversas fontes de variação foram calculados segundo HADDAD (1978).

\subsection{7 - Estimativas de variâncias das estimativas de con- trastes entre duas médias de tratamentos}

Förmulas para os cálculos das estimativas de variânclas das estimativas de contrastes entre duas médias de tratamentos, foram obtidas partindo-se da função linear de contrastes entre duas 
médias de tratamentos, onde em um deles ocorreram subparcelas perdi das ou em ambos, para os seguintes casos:

a) Duas médias de tratamentos $T$;

b) Duas médias de tratamentos T';

c) Duas médias de tratamentos $T^{\prime}$, dentro de um mesmo nÍvel de tratamento $T$.

A seguir, é apresentado o desenvolvimento teórico para o caso de duas subparcelas perdidas em tratamentos $T^{\text {e }} T^{\prime}$ diferentes, em que se deseja estimar a variància das estimativas de con trastes entre duas médias de tratamentos $T^{\prime}$, onde um deles perdeu uma subparcela. A estimativa da média de um tratamento T' qualquer, onde ocorreu a subparcela perdida, é dada por:

$$
\tilde{m}_{k_{1}}^{\prime}=\frac{T_{k_{1}}^{\prime}+x_{1}}{I J} \text {. }
$$

$$
\text { Substituindo-se na expressão }(4 \cdot 2.7 . a) x_{1} \text { pelo seu va }
$$

lor encontrado en (4.2.3.a), obteve-se,

$$
\hat{m}_{k_{1}}^{\prime}=\frac{1}{I J}\left[T_{k_{1}}^{\prime}+\frac{J P_{1}+K\left(T_{1} T_{k_{1}}^{\prime}\right)-T_{1}}{(J-1)(K-1)}\right]
$$

mas,

$$
\begin{aligned}
& T_{k_{1}}^{\prime}=\sum_{1}\left(T_{1} T_{k_{1}}^{\prime}\right) \\
& T_{1}=T_{1} T_{k_{1}}^{\prime}+P_{1}+M .
\end{aligned}
$$

onde, $\sum_{1}\left(T_{1} T_{k}^{\prime}\right)$ : somatório dos totais das subparcelas existentes que receberam tratamentos $T_{1}$ e $T_{k}^{\prime}$ corresponden- 
te à subparcela perdida $x_{1}$;

M: total das subparcelas existentes que receberam o tratamento $T_{i}$ que contêm a subparcela perdida, exceto as que pertencem a $P_{1}$ e $\left(T_{1} T_{k}{ }_{k}\right)$.

Ordenando-se convenientemente os termos semelhantes,

obteve-se,

$$
\hat{m}_{k_{1}}^{\prime}=\frac{1}{I J}\left[\frac{J}{J-1}\left(T_{1} T_{k_{1}}^{\prime}\right)+\frac{1}{k-1} P_{1}-\frac{1}{(J-1)(K-1)} M+\sum_{i}\left(T_{i} T_{k 1}^{\prime}\right)\right]
$$

logo,

$$
\begin{aligned}
& \nabla\left(\hat{m}_{k_{1}}^{\prime}\right)=\left[\frac{1}{I J}+\frac{K}{I^{2} J(J-1)(K-1)}\right] \quad \operatorname{QMR}(b) \\
& \text { Como, }
\end{aligned}
$$

$$
\nabla\left(\bar{m}_{k}^{\prime}\right)=\frac{1}{I J} \operatorname{QMR}(b)
$$

portanto,

$$
\hat{V}\left(\hat{m}_{k}^{\prime}-\hat{m}_{k 1}^{\prime}\right)=\frac{2}{I J}\left[1+\frac{k}{2 I(J-1)(K-1)}\right] \text { QMR(b) . }
$$


40.

\section{RESULTADOS E DISCUSSÃO}

Para melhor compreensão da metodologia proposta, foram apresentadas, separadamente, para cada caso estudado no delinea mento inteiramente casualizado com parcelas subdivididas, as estima tivas das observações perdidas, somas de quadrados ajustadas, corre ções "U", análise de variância e as estimativas de variàncias das estimativas de contrastes entre duas médias de tratamentos.

Aos termos já definidos, foram acrescentados os seguintes:

$T_{i} T_{k_{12}}$ : total das subparcelas existentes que receberam tratamentos $T_{i}$ e $T_{k}^{\prime}$ correspondente às subparcelas perdidas $x_{1} \in x_{2}$

$T_{k 12}$ : total das subparcelas existentes que receberam o tratamento $T_{k}^{\prime}$ correspondente às subparcelas perdidas $x_{1}$ e $x_{2}$. 


\section{1 - Caso de Uma Subparcela Perdida}

5.1.1 - Fórmula para estimativa de uma subparcela perdida $\left(x_{1}\right)$ e suas novas estimativas $\left(y_{1}, z_{1}\right)$ para utilização do método do residuo condicional

$$
\begin{aligned}
& x_{1}=\frac{J P_{1}+K\left(T_{i} T_{k_{1}}^{\prime}\right)-T_{i}}{(J-I)(K-1)} \\
& y_{1}=\frac{I J P_{1}+K T_{k_{1}}^{\prime}-G}{(I J-1)(K-1)} \\
& z_{1}=\frac{P_{1}}{K-1}
\end{aligned}
$$

Percebe-se que:

- A fórmula (5.1.1.a) é concordante com a apresentada por ANDERSON (1946), quando o autor aplicou o método da covariância;

- As fórmulas apresentadas em (5.1.1.a), (5.1.1.b) e (5.1.1.c) são idênticas às apresentadas por PINHO (1973), o que era esperado, pois, a segunda parte da análise de variância do delineamento estudado pela autora é a mesma que a deste trabalho.

\section{1 .2 - Somas de quadrados ajustadas e correção "U"}

A expressão da correção "U" para a soma de quadrados da interação $\operatorname{Tx}^{\prime}(x)^{\prime}$ é dada por:

$$
U=\frac{(I J-I)(K-1)}{I J K}\left(x_{1}-y_{1}\right)^{2}
$$


Esta fórmula é concordante com a apresentada por PINHO (1973), e verifica-se que a correção "U" será sempre positiva, portanto, a SQTXT'(x) estará sempre superestimada como afirmaram AN DERSON (1946), PINHO (1973), HADDAD (1978) e PIMENTEL GOMES (1978). A soma de quadrados de tratamentos $T^{\prime}$ ajustada foi obtida através da expressão dada em $(4.2 .4 . d)$.

\subsection{3 - Anālise de variância, para o caso de uma subparce- la perdida $\left(x_{513}\right)$}

Com os dados do experimento A (Tabela 12), procedeuse à análise de variância, cujos resultados são apresentados na Tabela 4 .

Tabela 4 - Análise de variància do experimento $A$, com somas de quadrados ( $S Q$ ) sem correção e corrigidas [SQ(C)] conforme o proposto neste trabalho, quando foi perdida uma subparce la $\left(x_{513}\right)$ 。

\begin{tabular}{lcccc}
\hline Fonte de Variação & G.L. & S.Q. & S.Q.(c) & Q.M.(c) \\
\hline Tratamentos T & 4 & 33,2643 & 33,2643 & $8,3161^{*}$ \\
Residuo (a) & 10 & 16,0589 & 16,0589 & 1,6059 \\
\hline Parcelas & 14 & 49,3232 & 49,3232 & \\
\hline Tratamentos T' & 3 & 21,5291 & 21,2757 & $7,0919^{* *}$ \\
Interaçöes TXT' & 12 & 19,9871 & 19,8186 & $1,6516 n s$ \\
Residuo (b) & 29 & 45,0649 & 45,0649 & 1,5540 \\
\hline Total & 58 & & 135,4824 & \\
\hline
\end{tabular}

* : significativo ao nível de $5 \%$ de probabilidade;

**: significativo ao nível de $1 \%$ de probabilidade;

ns: não significativo ao nível de $5 \%$ de probabilidade;

(1): os quadrados médios $[Q M(c)]$ foram obtidos a partir das $S Q(c)$. 
Analisando-se a Tabela 4, vê-se que a $\operatorname{SQT}^{\prime} T^{\prime}(x)$ e SQT' $(x)$ estão superestimadas, como afirmaram ANDERSON (1946), PINHD (1973), HADDAD (1978) E PIMENTEL GOMES (1978)。

As estimativas de variâncias das estimativas de contrastes entre duas médias de tratamentos. são calculadas através das förmulas apresentadas em 5.1.4, para aplicação de Testes de Signifi cância.

5.1 .4 - Estimativas de variâncias das estimativas de contrastes entre duas médias de tratamentos

5.1.4.1 - Caso de duas médias de tratamentos $T$, on de um deles perdeu uma subparcela

$$
V\left(\hat{m}_{1}-\hat{m}_{1}\right)=\left(\frac{1}{J K}+\frac{1}{J K-1}\right\} \operatorname{QMR}(a)
$$

5.1.4.2 - Caso de duas médias de tratamentos $T^{\prime}$, on de um deles perdeu uma subparcela

$$
\begin{aligned}
V\left(\hat{m}_{k}^{\prime}-\hat{m}_{k l}^{\prime}\right)= & \frac{2}{I J}\left[1+\frac{K}{2 I(J-I)(K-I)}\right] \text { QMR(b) } \\
5.1 .4 .3- & \text { Caso de duas médias de tratamentos } T^{\prime}, \\
& \text { dentro de um mesmo nivel do tratamento } \\
& T \text {, onde um deles perdeu uma subparcela }
\end{aligned}
$$

$$
V\left[\left(\hat{m}_{k}^{3}-\hat{m}_{k l}^{i}\right) / T_{i}\right]=\frac{2}{J}\left[1+\frac{k}{2(J-1)(K-1)}\right] \text { QMR }(b)
$$


Nota-se que:

- A estimativa da variância em 5.1.4.1 corresponde à estimativa de variância das estimativas de contrastes entre duas médias de tratamentos $T$ com número diferente de repetições;

- As estimativas das variâncias em 5.1 .4 .2 e 5.1 .4 .3 são concordantes com as apresentadas por ANDERSON (1946) e PINHO (1975);

- A estimativa da variância em 5.1.4.3 é discordante da apresentada por COCHRAN e COX (1976),

\section{2 - Caso de Duas Subparcelas Perdidas fluma Mesma Parcela Com-} posta de $k>2$ Subparcelas

5.2.1 - Förmulas para estimativas de duas subparcelas perdidas $\left(x_{1}, x_{2}\right)$ e suas novas estimativas $\left(y_{1}, y_{2}\right.$, $z_{1}, z_{2}$ ) para utilização do método do resíduo condicional

$$
\begin{aligned}
& x_{1}=\frac{J P_{1}+(K-I)\left(T_{i} T_{k_{1}}^{\prime}\right)+\left(T_{i} T_{k_{2}}^{\prime}\right)-T_{1}}{(J-1)(K-2)} \\
& x_{2}=\frac{J P_{1}+(K-1)\left(T_{i} T_{k_{2}}^{\prime}\right)+\left(T_{i} T_{k_{1}}^{\prime}\right)-T_{i}}{(J-I)(K-2)} \\
& y_{1}=\frac{I J P_{1}+(K-1) T_{k_{1}}^{\prime}+T_{k_{2}}-G}{(I J-1)(K-2)} \\
& y_{2}=\frac{I J P_{1}+(K-1) T_{k_{2}}^{\prime}+T_{k_{1}}-G}{(I J-1)(K-2)} \\
& z_{1}=z_{2}=\frac{P_{1}}{K-2}
\end{aligned}
$$


Verifica-se que estas fórmulas são concordantes com aquelas apresentadas por PINHO (1973), pelas mesmas razões descritas em 5.1.1.

\subsection{2 - Somas de quadrados ajustadas e correção " $U$ "}

A expressão da correção "U" para a soma de quadrados da interação Ty.'(x) é dada por:

$$
\begin{aligned}
U= & \frac{(I J-I)(K-1)}{I J K} \cdot\left[\left(x_{1}-y_{1}\right)^{2}+\left(x_{2}-y_{2}\right)^{2}\right]- \\
& -\frac{2(I J-1)}{I J K}\left(x_{1}-y_{1}\right)\left(x_{2}-y_{2}\right)
\end{aligned}
$$

Analisando-se esta fórmula, verifica-se que a correção "U" seră sempre positiva, portanto, a $\operatorname{SQT} T \times T^{\prime}(x)$ estará sempre superestimada, concordando com ANDERSON (1946), PINHO (1973), HADDAD (1978) e PIMENTEL GOMES (1978).

A soma de quadrados de tratamentos T' ajustada foi obtida através da expressão dada em $(4.2 .4 . d)$.

5.2.3 - Anälise de variância, para o caso de duas subparce las perdidas $\left(x_{321}, x_{322}\right)$ numa mesma parcela composta de $k>2$ subparcelas

Com os dados do experimento A (Tabela 12), procedeuse à anālise de variância, cujos resultados são apresentados na Tabela 5. 
Tabela 5 - Análise de variância do experimento $A$, com somas de quadrados (SQ) sem correção e corrigidas [SQ(c)] conforme o proposto neste trabalho, quando foram perdidas duas subparcelas $\left(x_{321}, x_{322}\right)$ numa mesma parcela.

\begin{tabular}{lcccc}
\hline Fonte de Variação & G.L. & S.Q. & S.Q.(c) & Q.M.(c) \\
\hline Tratamentos T & 4 & 30,9010 & 30,9010 & $7,7252^{* 3}$ \\
Residuo (a) & 10 & 18,1081 & 18,1081 & 1,8108 \\
\hline Parcelas & 14 & 49,0091 & 49,0091 & \\
\hline Tratamentos T' & 3 & 14,8728 & 15,8742 & $5,2914 *$ \\
Interação TxT' & 12 & 22,6553 & 21,5020 & $1,7918 n$ ( \\
Residuo (b) & 28 & 40,1638 & 40,1638 & 1,4344 \\
\hline Total & 57 & & 126,5491 & \\
\hline
\end{tabular}

Observando-se a Tabela 5, verifica-se que:

- A SQTXT'(x) está superestimada como afirmaram ANDERSON(1946). PINHO (1973), HAODAD (1978) E PIMENTEL GOMES (1978);

- A SQT' $(x)$ está subestimada, o que concorda com PINHO (1973), pois a autora afirmou que a $\operatorname{SQT}^{\prime}(x)$ pode estar subestimada.

As estimativas de variâncias das estimativas de contrastes entre duas médias de tratamentos são calculadas através das förmulas apresentadas em 5.2.4, para aplicação de Testes de Significância. 
5.2.4 - Estimativas de variancias das estimativas de contrastes entre duas médias de tratamentos

5.2.4.1 - Caso de duas médias dos tratamentos $T$, on de um deles perdeu duas subparcelas

$$
\hat{V}\left(\hat{m}_{i}-\hat{m}_{1},\right)=\left(\frac{1}{J K}+\frac{1}{J K-2}\right\} \operatorname{QMR}(a)
$$

5.2.4.2 - Caso de duas médias de tratamentos T', onde um deles perdeu uma subparcela

$$
\nabla\left(\hat{m}_{k}^{\prime}-\hat{m}_{k I}^{\prime}\right)=\frac{2}{I J}\left[1+\frac{k-I}{2 I(J-I)(K-2)}\right] \operatorname{QMR}(b)
$$

5.2.4.3 - Caso de duas médias de tratamentos T', onde cada um perdeu uma subparcela

$$
\hat{V}\left(\hat{m}_{k_{1}}^{\prime}-\hat{m}_{k_{2}}^{\prime}\right)=\frac{2}{I J}\left[1+\frac{1}{I(J-I)}\right] \operatorname{QMR}(b)
$$

5.2.4.4 - Caso de duas mëdias de tratamentos $T^{\prime}$, dentro de um mesmo nível de tratamento $T$, onde um deles perdeu uma subparcela

$$
\left.\nabla_{L}^{[}\left(\hat{m}_{k}^{\prime}-\hat{m}_{k 1}^{\prime}\right) / T_{i}\right]=\frac{2}{J}\left[1+\frac{k-1}{2(j-1)(k-2)}\right] \operatorname{QMR}(b)
$$




\subsubsection{5 - Caso de duas médias de tratamentos $T^{\prime}$, dentro de um mesmo nível de tratamento $T$. onde cada um perdeu uma sutparcela}

$$
\hat{V}\left[\left(\hat{m}_{k_{1}}^{\prime}-\hat{m}_{k_{2}}^{\prime}\right) / T_{i}\right]=\frac{2}{J-1} \text { QMR(b). }
$$

\section{Nota-se que:}

- A estimativa da variância em 5.2.4.1 corresponde à estimativa de variância das estimativas de contrastes entre duas médias de tratamentos $T$ com número diferente de repetições;

- A estimativa da variância em 5.2.4.5 é idêntica àquela apresentada por HADDAD (1978), o que era esperado, pois, a contribuição da única subparcela restante na parcela onde foram perdidas as subparcelas é nula, quanto aos graus de liberdade.

\section{3 - Caso de Duas Subparcelas Perdidas que Receberam Tratamen- tos $T$ e $T^{\prime}$ Diferentes}

5.3.1 - Förmulas para estimativas de duas subparcelas perdidas $\left(x_{1}, x_{2}\right)$ e suas novas estimativas $\left(y_{1}, y_{2}\right.$, $z_{1}, z_{2}$ ) para utilização do método do resíduo con dicional

$$
\begin{aligned}
& x_{1}=\frac{J P_{1}+K\left(T_{1} T_{k_{1}}^{\prime}\right)-T_{1}}{(J-I)(K-I)} \\
& x_{2}=\frac{J P_{2}+K\left(T_{1}, T_{k_{2}}^{\prime}\right)-T_{i}}{(J-1)(K-1)}
\end{aligned}
$$




$$
\begin{gathered}
y_{1}=\frac{(I J-1)(K-I)\left(I J P_{1}+K T_{K_{1}}^{\prime}-G\right)-I J P_{2}-K T_{k_{2}}+G}{(I J-I)^{2}(K-1)^{2}-1} \\
y_{2}=\frac{(I J-1)(K-1)\left(I J P_{2}+K T_{K_{2}}^{\prime}-G\right)-I J P_{1}-K T_{K_{1}}^{\prime}+G}{(I J-1)^{2}(K-1)^{2}-1} \\
z_{1}=\frac{P_{1}}{K-1} \\
z_{2}=\frac{P_{2}}{K-1}
\end{gathered}
$$

Vê-se que estas fórmulas são concordantes com aquelas apresentadas por PINHO (1973), pelas mesmas razões descritas em 5.1 .1 .

\section{3 .2 - Somas de quadrados ajustadas e correção "U"}

A expressão da correção "U" para a soma de quadrados da interação $\operatorname{Tx}^{\prime}(x)$ é dada por:

$$
\begin{aligned}
U= & \frac{(I J-I)(K-1)}{I J K} \cdot\left[\left(x_{1}-y_{1}\right)^{2}+\left(x_{2}-y_{2}\right)^{2}\right]+ \\
& +\frac{2}{I J K}\left(x_{1}-y_{1}\right)\left(x_{2}-y_{2}\right)
\end{aligned}
$$

Examinando-se esta fórmula, percebe-se que a correção "U" será sempre positiva, portanto, a $\mathrm{SQTXT}^{\prime}[x]$ estará sempre superestimada, como afirmaram ANDERSON (1946), PINHO (1973), HADDAD (1978) E PIMENTEL GOMES (1978). 
A soma de quadrados de tratamentos T' ajustada foi obtida através da expressão dada em $(4.2 .4 . d)$.

\section{3 .3 - Análise de variância, para o caso de duas subparce las perdidas $\left(x_{222}, x_{313}\right)$ que receberam tratamentos $T$ e $T^{\prime}$ diferentes}

Com os dados do experimento A (Tabela 12), procedeu-se à análise de variãncia, cujos resultados são apresentados na Ta bela 6 .

Tabela 6 - Análise de variáncia do experimento A, com somas de quadrados (SQ) sem correção e corrigidas [SQ(c)] conforme o proposto neste trabalho, quando foram perdidas duas subparcelas $\left(x_{222}\right.$ e $\left.x_{313}\right)$ que receberam tratamentos $T$ e T' diferentes.

\begin{tabular}{lcccc}
\hline Fonte de Variação & G.L. & S.Q. & S.Q.(c) & Q.M. (c) \\
\hline Tratamentos T & 4 & 28,0002 & 28,0002 & $7,0000^{*}$ \\
Resíduo (a) & 10 & 17,0571 & 17,0571 & 1,7057 \\
\hline Parcelas & 14 & 45,0573 & 45,0573 & \\
\hline Tratamentos T' & 3 & 18,6910 & 17,3615 & $5,7872^{*}$ \\
Interação TxT' & 12 & 21,3902 & 20,8469 & $1,7372 n$ s \\
ResIduo (b) & 28 & 46,9965 & 46,9965 & 1,6784 \\
\hline Total & 57 & & 130,2622 & \\
\hline
\end{tabular}

Analisando-se a Tabela 6, verifica-se que as SQT'( $x)$ e SQTTXT'(x) estão superestimadas, como afirmaram ANOERSON (1946), PINHO (1973), HADDAD (1978) e PIMENTEL GOMES (1978). 
As estimativas de variàncias das estimativas de contrastes entre duas médias de tratamentos, são calculadas através das fórmulas apresentadas em 5.3.4, para aplicação de Testes de Signifi cància.

5.3.4 "Estimativas de variâncias das estimativas de con" trastes entre duas médias de tratamentos

5.3.4.1 - Caso de duas médias de tratamentos $T$, on de um deles perdeu uma subparcela

$$
V\left(\hat{m}_{i}-\hat{m}_{i^{\prime}}\right)=\left(\frac{1}{J K}+\frac{1}{J K-1}\right) \operatorname{QMR}(a)
$$

5.3.4.2 - Caso de duas médias de tratamentos $T$, onde cada um perdeu uma subparcela

$$
\begin{gathered}
V\left(\hat{m}_{i}-\hat{m}_{i^{\prime}}\right)=\frac{2}{J K-1} \operatorname{QMR}(a) \\
5.3 .4 .3-\text { Caso de duas médias de tratamentos } T^{\prime} \\
\text { onde um deles perdeu uma subparcela } \\
\nabla\left(\hat{m}_{k}^{\prime}-\hat{m}_{k_{1}}^{\prime}\right)=\frac{2}{I J}\left[1+\frac{K}{2 I(J-I)(K-I)}\right] \operatorname{QMR}(b)
\end{gathered}
$$




$$
\begin{aligned}
& \text { 5.3.4.4 - Caso de duas médias de tratamentos } T^{\prime} \text {, } \\
& V\left(\tilde{m}_{k_{1}}^{\prime}-\hat{m}_{k_{2}}^{\prime}\right)=\frac{2}{I J}\left[1+\frac{k}{I(J-1)(K-1)}\right] \quad \operatorname{QMR}(b) \\
& \text { 5.3.4.5 - Caso de duas médias de tratamentos T', } \\
& \nabla\left[\left(\hat{m}_{k}^{\prime}-\hat{m}_{k l}^{\prime}\right) / T_{i}\right]=\frac{2}{J}\left[1+\frac{k}{2(J-1)(k-1)}\right] \operatorname{QMR}(b) \\
& \text { Verifica-se que a estimativa da variância em 5.3.4.1 }
\end{aligned}
$$
ções.

5.4 - Caso de Duas Subparcelas Perdidas que Receberam Tratamentos $T$ Diferentes e Mesmo Tratamento $T^{\prime}$

5.4.1 - Förmulas para estimativas de duas subparcelas perdidas $\left(x_{1}, x_{2}\right)$ e suas novas estimativas $\left(y_{1}, y_{2}\right.$, $z_{1}, z_{2}$ ) para utilização do método do resíduo condicional

$$
x_{1}=\frac{J P_{1}+K\left(T_{i} T_{k 1}\right)-T_{i}}{(J-1)(K-1)}
$$


53.

$$
\begin{aligned}
& x_{2}=\frac{J P_{2}+K\left(T_{i} \cdot T_{k_{2}}\right)-T_{i^{\prime}}}{(J-I)(K-I)} \\
& y_{1}=\frac{(I J-I) P_{1}+P_{2}+K T_{k_{1}}^{\prime}-G}{(I J-2)(K-1)} \\
& y_{2}=\frac{(I J-1) P_{2}+P_{1}+K T_{k_{1}}^{\prime}-G}{(I J-2)(K-I)} \\
& z_{1}=\frac{P_{1}}{K-1} \\
& z_{2}=\frac{P_{2}}{K-1}
\end{aligned}
$$

Vê-se que estas fórmulas são concordantes com aquelas apresentadas por PINHO (1973), pelas mesmas razões descritas em 5.1 .1

\section{4 .2 - Somas de quadrados ajustadas e correçāo "un"}

A expressão da correção "U" para a soma de quadrados da interação $\operatorname{TXT}^{\prime}(x)$ é dada por:

$$
\begin{aligned}
U= & \frac{(I J-I)(K-I)}{I J K}\left[\left(x_{1}-y_{1}\right)^{2}+\left(x_{2}-y_{2}\right)^{2}\right]- \\
& -\frac{2(K-I)}{I J K}\left(x_{1}-y_{1}\right)\left(x_{2}-y_{2}\right)
\end{aligned}
$$

Observando-se esta fórmula, verifica-se que a correção "U" será sempre positiva, portanto, a $\mathrm{SQT}_{\mathrm{T}} \mathrm{T}^{\prime}(\mathrm{x})$ estará sempre 
superestimada, concordando com ANDERSON (1946), PINHO (1973), HADDAD (1978) e PIMENTEL GOMES (1978).

A soma de quadrados de tratamentos $T$ ' ajustada foi obtida através da expressão dada em $(4.2 .4 . d)$.

5.4 .3 - Anälise de variância, para o caso de duas subparce las perdidas $\left(x_{111}, x_{221}\right)$ que receberam tratamentos $T$ diferentes e mesmo tratamento $T^{\prime}$

Com os dados do, experimento A (Tabela. 12), procedeu-se à anālise de variância, cujos resultados são apresentados na Tabela 7.

Tabela 7 - Anälise de variância do experimento $A$ o com somas de quadrados (SQ) sem correção e corrigidas [SQ(c)] conforme o proposto neste trabalho, quando foram perdidas duas subparcelas $\left(x_{113}, x_{221}\right)$ que receberam tratamentos $T$ diferentes e mesmo tratamento $T$ '。

\begin{tabular}{lcccc}
\hline Fonte de Variação & G.L. & S.Q. & S.Q.(c) & Q.M.(c) \\
\hline Tratamentos T & 4 & 28,5328 & 28,5328 & $7,1332^{*}$ \\
Residuo (a) & 10 & 15,5624 & 15,5624 & 1,5562 \\
\hline Parcelas & 14 & 44,0952 & 44,0952 & \\
\hline Tratamentos T' & 3 & 18,7753 & 20,3931 & $6,7977^{*}$ \\
Interação TXT' & 12 & 22,0330 & 20,3268 & $1,6939 n s$ \\
Resíduo (b) & 28 & 51,1210 & 51,1210 & 1,8258 \\
\hline Total & 57 & & 135,9361 & \\
\hline
\end{tabular}




\section{Examinando-se a Tabela 7, percebe-se que:}

- A SQTXT'(x) está superestimada, como afirmaram ANDERSON(1946), PINHO (1973), HADDAD (1978) e PIMENTEL GOMES (1978):

- A SQT' $(x)$ está subestimada, o que concorda com PINHO (1973), pois, a autora afirmou que a $\operatorname{SQT}^{\prime}(x)$ pode estar subestimada.

As estimativas de variâncias das estimativas de contrastes entre duas mëdias de tratamentos, são calculadas através das förmulas apresentadas em 5.4.4, para aplicação de Testes de Signifi cância.

5.4.4 - Estimativas de variâncias das estimativas de contrastes entre duas médias de tratamentos

5.4.4.1 - Caso de duas médias de tratamentos $T$, on de um deles perdeu uma subparcela

$$
V\left(\hat{m}_{i}-\tilde{m}_{i}\right)=\left(\frac{1}{J K}+\frac{1}{J K-1}\right) \operatorname{QMR}(a)
$$

5.4 .4 .2 - Caso de duas médias de tratamentos $T^{\prime}$, onde um deles perdeu duas subparcelas

$$
O\left(\hat{m}_{k}^{\prime}-\hat{m}_{k_{1}}^{\prime}\right)=\frac{2}{I J}\left[1+\frac{K}{I(J-1)(K-1)}\right] \quad \operatorname{QMR}(b)
$$




\subsubsection{3 - Caso de duas médias de tratamentos $T^{\prime}$, dentro de um mesmo nível de tratamento $T$, onde um deles perdeu uma subparcela}

$$
\nabla\left[\left(\hat{m}_{k}^{\prime}-\hat{m}_{k 1}^{\prime}\right) / T_{i}\right]=\frac{2}{J}\left[1+\frac{k}{2(J-1)(k-1)}\right] \operatorname{QMR}(b)
$$

Verifica-se que a estimativa da variāncia em 5.4.4.I corresponde à estimativa de variância da estimativa de contraste en tre duas médias de tratamentos $T$ com número diferente de repetições.

\section{5 - Caso de Duas Subparcelas Perdidas que Receberam Mesmo Tra- tramento $T$ e Tratamentos $T^{\prime}$ Diferentes, em Parcelas Distin tas}

5.5.1 Förmulas para estimativas de duas subparcelas perdidas $\left(x_{1}, x_{2}\right)$ e suas novas estimativas $\left(y_{1}, y_{2}\right.$, $z_{1}, z_{2}$ ) para utilização do método do resíduo condicional

$$
\begin{aligned}
& x_{1}=\frac{(J-1)(K-1)\left[J P_{1}+K\left(T_{i} T_{k 1}^{\prime}\right)-T_{i}\right]-J P_{2}-K\left(T_{i} T_{k_{2}}^{\prime}\right)+T_{i}}{(J-I)^{2}(K-I)^{2}-1} \\
& x_{2}=\frac{(J-1)(K-1)\left[J P_{2}+K\left(T_{1} T_{k_{2}}^{\prime}\right)-T_{i}\right]-J P_{1}-K\left(T_{i} T_{k_{1}}^{\prime}\right)+T_{i}}{(J-I)^{2}(K-I)^{2}-1} \\
& y_{1}=\frac{(I J-1)(K-1)\left(I J P_{1}+K T_{k_{1}}^{\prime}-G\right)-I J P_{2}-K T_{k_{2}}^{\prime}+G}{(I J-I)^{2}(K-I)^{2}-1} \\
& y_{2}=\frac{(I J-I)(K-I)\left(I J P_{2}+K T_{K_{2}}-G\right)-I J P_{1}-K T_{k_{1}}+G}{(I J-I)^{2}(K-I)^{2}-1}
\end{aligned}
$$




$$
\begin{aligned}
& z_{1}=\frac{P_{1}}{K-1} \\
& z_{2}=\frac{P_{2}}{K-1}
\end{aligned}
$$

Nota-se que estas fórmulas são concordantes com aque las apresentadas por PINHO (1973), pelas mesmas razões descritas em 5.1 .1$.

\section{5 .2 - Somas de quadrados ajustadas e correção "U"}

A expressão da correção "U" para a soma de quadrados da interação $T_{X} T^{\prime}(x)$ é dada por:

$$
\begin{aligned}
U= & \frac{(I J-1)(K-1)}{I J K}\left[\left(x_{1}-y_{1}\right)^{2}+\left(x_{2}-y_{2}\right)^{2}\right]+ \\
& +\frac{2}{I J K}\left(x_{1}-y_{1}\right)\left(x_{2}-y_{2}\right)
\end{aligned}
$$

Examinando-se esta fórmula, verifica-se que a correção "U" será sempre positiva, portanto, a SQTXT'(x) estará sempre superestimada, como afirmaram ANDERSON (1946), PINHO (1973), HADDAD (1978) \& PIMENTEL GOMES (1978).

A soma de quadrados de tratamentos T' ajustada foi obtida através da expressão dada em $(4.2 .4 . d)$. 
5.5.3 - Anälise de variância, para o caso de duas subparce las perdidas $\left(x_{522}, x_{531}\right)$ que receberam mesmo tratamento $T$ e tratamentos $T^{\prime}$ diferentes, em parcelas distintas

Com os dados do experimento A (Tabela 12), procedeu-se à análise de variância, cujos resultados são apresentados na Ta bela 8 .

Tabela 8 - Análise de variáncia do experimento $A$, com somas de quadrados (SQ) sem correção e corrigida $[S Q(c)]$ conforme o proposto neste trabalho, quando foram perdidas duas subparcelas $\left(x_{522}, x_{531}\right)$ que receberam mesmo tratamento $T e$ tratamentos $T$ ' diferentes, em parcelas distintas.

\begin{tabular}{lcccc}
\hline Fonte de Variação & G.L. & S.Q. & S.Q.(c) & Q.M. (c) \\
\hline Tratamentos T & 4 & 27,9978 & 27,9978 & $6,9994^{*}$ \\
Residuo (a) & 10 & 15,9090 & 15,9090 & 1,5909 \\
\hline Parcelas & 14 & 43,9068 & 43,9068 & \\
\hdashline Tratamentos T' & 3 & 9,3289 & 9,3210 & $3,1070 n s$ \\
Interação TxT' & 12 & 15,8696 & 15,0278 & $1,2523 n s$ \\
Residduo (b) & 28 & 37,3528 & 37,3528 & 1,3340 \\
\hline Total & 57 & & 105,6084 & \\
\hline
\end{tabular}

Analisando-se a Tabela 8 , verifica-se que as $\operatorname{SQT}^{\prime}(x)$ e SQTXT'(x) estão superestimadas, como afirmaram ANDERSON (1946), PI NHO (1973), HADDAD (1978) E PIMENTEL GOMES (1978).

As estimativas de variâncias das estimativas de con 
trastes entre duas médias de tratamentos são calculadas através das fórmulas apresentadas em 5.5.4, para aplicação de Testes de Signifí cância.

5.5.4 - Estimativas de variäncias das estimativas de contrastes entre duas mëdias de tratamentos

5.5 .4 .1 - Caso de duas mëdias de tratamentos $T$, on de um deles perdeu duas subparcelas

$$
\hat{V}\left(\hat{m}_{i}-\hat{m}_{i},\right)=\left(\frac{1}{J K}+\frac{1}{J K-2}\right\} \operatorname{QMR}(a)
$$

5.5.4.2 - Caso de duas mëdias de tratamentos $T^{\prime}$, onde um deles perdeu uma subparcela

$$
V\left(\hat{m}_{k}^{\prime}-\hat{m}_{k i}^{\prime}\right)=\frac{2}{I J}\left\{1+\frac{K(J-1)(K-1)}{2 I\left[(J-1)^{2}(K-I)^{2}-1\right]}\right\} \operatorname{QMR}(b)
$$

5.5.4.3 - Caso de duas médias de tratamentos $T^{\prime}$, onde cada um deles perdeu uma subparcela

$$
\begin{aligned}
& Q\left(\hat{m}_{k 1}^{\prime}-\hat{m}_{k 2}^{\prime}\right)=\frac{2}{I J}\left\{1+\frac{k}{I[(J-1)(k-1)-1]}\right\} \operatorname{QMR}(b) \\
& V\left[\left(\hat{m}_{k}^{\prime}-\hat{m}_{k 1}^{\prime}\right) / T_{i}\right]=\frac{2}{I J}\left\{1+\frac{K(J-1)(K-1)}{2\left[(J-1)^{2}(K-1)^{2}-1\right]}\right\} \operatorname{QMR}(b)
\end{aligned}
$$




\subsubsection{5 - Caso de duas mëdias de tratamentos $T^{\prime}$, dentro de um mesmo nivel de tratamento $T$, onde cada um perdeu uma subparcela}

$$
V\left[\left(\hat{m}_{k_{1}}^{\prime}-\hat{m}_{k_{2}}^{\prime}\right) / T=1\right]=\frac{2}{J}\left[1+\frac{k}{(J-1)(k-1)-1}\right] \text { QMR }(b)
$$

Vê-se que a estimativa da variância em $5.5 .4 .1 \mathrm{cor}-$ responde à estimativa da variância da estimativa de contraste entre duas médias de tratamentos $T$, com número diferente de repetições.

\section{6 - Caso de Duas Subparcelas Perdidas que Receberam Mesmos Tra tamentos $T^{\text {e }} \mathrm{T}^{\prime}$}

5.6.1 - Fórmulas para estimativas de duas subparcelas perdidas $\left(x_{1}, x_{2}\right)$ e suas novas estimativas $\left(y_{1}, y_{2}\right.$, $z_{1}, z_{2}$ ) para utilização do mëtodo do resíduo condicional

$$
\begin{aligned}
& x_{3}=\frac{(J-1) P_{1}+P_{2}+K\left(T_{i} T_{K_{12}}^{\prime}\right)-T_{i}}{(J-2)(K-I)} \\
& x_{2}=\frac{(J-1) P_{2}+P_{1}+K\left(T_{i} T_{K_{12}}^{\prime}\right)-T_{1}}{(J-2)(K-I)} \\
& y_{1}=\frac{(I J-1) P_{1}+P_{2}+K T_{K_{12}}^{\prime}-G}{(I J-2)(K-1)} \\
& y_{2}=\frac{(I J-1) P_{2}+P_{1}+K T_{K_{12}}-G}{(I J-2)(K-1)}
\end{aligned}
$$


61.

$$
\begin{aligned}
& z_{1}=\frac{P_{1}}{k-1} \\
& z_{2}=\frac{P_{2}}{k-1}
\end{aligned}
$$

Nota-se que estas fórmulas são concordantes com aque las apresentadas por PINHO (1973), pelas mesmas razões descritas em 5.1 .1$.

\subsection{2 - Somas de quadrados ajustadas e correção "U"}

A expressão da correção "U" para a soma de quadrados da interação $\operatorname{Tx}^{\prime}(x)$ é dada por:

$$
U=\frac{2(I J-1)(K-I)}{I J K}\left(x_{1}-y_{1}\right)^{2} .
$$

Analisando-se esta fórmula, verifica-se que a correção "U" será sempre positiva, portanto, a SQTXT'(x) estará sempre superestimada, como afirmaram ANDERSON (1946), PINHO (1973), HADDAD (1978) \& PIMENTEL GOMES (1978).

A soma de quadrados de tratamentos T' ajustada foi obtida através da expressão dada em $(4.2 .4, d)$. 
62.

5.6.3 - Anālise de variância, para o caso de duas subparce las perdidas $\left(x_{514}, x_{554}\right)$, que receberam mesmos tra tamentos $T$ e $T^{\prime}$

Com os dados do experimento A (Tabela 12), procedeu-se à anālise de variância, cujos resultados são apresentados na Ta bela 9 .

Tabela 9 - Anälise de variância do experimento $A$, com somas de quadrados (SQ) sem correção e corrigidas [SQ(c)], conforme o proposto neste trabalho, quando foram perdidas duas subparcelas $\left(x_{514}, x_{554}\right)$ que receberam mesmos tratamentos $T$ e T'。

\begin{tabular}{lcccc}
\hline Fonte de Variação & G.L. & S.Q. & S.Q.(c) & Q.M.(c) \\
\hline Tratamentos $T$ & 4 & 27,8176 & 27,8176 & $6,9544^{*}$ \\
Residuo (a) & 10 & 16,8167 & 16,8167 & 1,6817 \\
\hline Parcelas & 14 & 44,6343 & 44,6343 & \\
\hline Tratamentos T' & 3 & 20,4342 & 20,4081 & $6,8027^{*}$ \\
Interação TxT' & 12 & 30,1430 & 23,3424 & $1,9452 n s$ \\
Resíduo (b) & 28 & 46,6145 & 46,6145 & 1,6648 \\
\hline Total & 57 & & 134,9993 & \\
\hline
\end{tabular}

Examinando-se a Tabela 9, verifica-se que as SQT'(x)

e SQTXT'(x) estão superestimadas, como afirmaram ANDERSON (1946), PI NHD (1973), HADDAD (1978) e PIMENTEL GOMES (1978).

As estimativas de variàncias das estimativas de contrastes entre duas médias de tratamentos, são calculadas através das 
63.

fórmulas apresentadas em 5.6.4, para aplicação de Testes de Signifi cância.

5.6.4 - Estimativas das variâncias das estimativas de contrastes entre duas mëdias de tratamentos

5.6.4.1 - Caso de duas médias de tratamentos $T$, on de um deles perdeu duas subparcelas

$$
\nabla\left(\tilde{m}_{i}-\tilde{m}_{i},\right)=\left(\frac{1}{J K}+\frac{1}{J K-2}\right) \operatorname{QMR}(a)
$$

5.6.4.2 - Caso de duas médias de tratamentos T', onde um deles perdeu duas subparcelas

$$
V\left(\hat{m}_{k}^{\prime}-\hat{m}_{k 12}^{\prime}\right)=\frac{2}{I J}\left[1+\frac{k}{I(J-2)(K-1)}\right] \operatorname{QMR}(b)
$$

5.6.4.3 - Caso de duas médias de tratamentos $T^{\prime}$, dentro de um mesmo nivel de tratamento $T$, onde um deles perdeu duas subparcelas

$$
O\left[\left(\hat{m}_{k}^{\prime}-\hat{m}_{k 12}^{\prime}\right) / T_{i}\right]=\frac{2}{J}\left[1+\frac{k}{(J-2)(k-1)}\right] \operatorname{QMR}(b)
$$

Verifica-se que a estimativa da variāncia em 5.6.4.1 corresponde à estimativa da varıanncia de contraste entre duas médias dos tratamentos $T$, com número diferente de repetições. 
5.7 - Caso de Uma Parcela Perdida, Composta de Duas Subparcelas

5.7.1 - Förmulas para estimativas de duas subparcelas perdidas $\left(x_{1}, x_{2}\right)$ e suas novas estimativas $\left(y_{1}, y_{2}\right.$, $z_{1}, z_{2}$ ) para utilização do método do resíduo condicional

$$
\begin{aligned}
& x_{1}=\frac{\left(T_{i} T_{k_{1}}^{\prime}\right)}{J-1} \\
& x_{2}=\frac{\left(T_{i} T_{k_{2}}^{\prime}\right)}{J-1} \\
& y_{1}=\frac{(J-1)\left(2 T_{k_{1}}^{\prime}-G\right)+(I J-1) T_{i}}{2(I J-1)(J-1)} \\
& y_{2}=\frac{(J-1)\left(2 T_{k_{2}}^{\prime}-G\right)+(I J-1) T_{i}}{2(I J-I)(J-1)} \\
& z_{1}=z_{2}=\frac{T_{i}}{2(J-1)}
\end{aligned}
$$

5.7.2 - Correção "U": para a soma de quadrados da interação. $\operatorname{TxT}^{\prime}(x)$

A expressão da correção "U" para a soma de quadrados da interação $\operatorname{Tx}^{\prime}(x)$ é dada por:

$$
U=\frac{I J-1}{I J}\left[\left(x_{1}-y_{1}\right)^{2}+\left(x_{2}-y_{2}\right)^{2}\right]
$$

Observando-se esta fórmula, verifica-se que a correção "U" será sempre positiva, portanto, a $\operatorname{SQTXT}(x)$ estará sempre 
superestimada, como afirmaram ANDERSON (1946), PINHO (1973), HADDAD (1978) \& PIMENTEL GOMES (1978).

\subsection{3 - Análise de variância, para o caso de uma parcela perdida, constituida de duas subparcelas $\left(x_{111}\right.$, $\left.x_{112}\right)$}

Com os dados do experimento B (Tabela 13), procedeu-se à análise de variância, cujos resultados são apresentados na Tạ bela 10.

Tabela 10 - Análise de variáncia do experimento $B$, com somas de qua drados (SQ) sem correção e corrigidas $[S Q(C)]$ conforme o proposto neste trabalho, quando ocorreu perda de uma parcela, constituída de duas subparcelas $\left(x_{111}, x_{112}\right)$.

\begin{tabular}{lcccc}
\hline Fonte de Variação & G.L. & S.Q. & S.Q.(c) & Q.M.(c) \\
\hline Tratamentos T & 1 & 61,2274 & 61,2274 & $61,2274 \mathrm{~ns}$ \\
Residuo (a) & 17 & 411,7731 & 411,7731 & 24,2219 \\
\hline Parcelas & 18 & 473,0005 & 473,0005 & \\
\hline Tratamentos T' & 1 & 10222,2299 & 9527,4445 & $9527,4445^{\star *}$ \\
Interação TXT' & 1 & 333,1855 & 315,6494 & $315,6494^{*}$ \\
Residuo (b) & 16 & 412,2111 & 412,2111 & 25,7632 \\
\hline Total & & & 10728,3055 & \\
\hline
\end{tabular}

Analisando-se a Tabela 10, verifica-se que as $\operatorname{SQT}^{\prime}(x)$ e $\operatorname{SQ̣TXT}(x)$ estão superestimadas, como afirmaram ANDERSON (1946), PI NHO (1973), HAODAD (1978) e PIMENTEL GOMES (1978). 
Pelos resultados da análise de variância, pode-se di zer que não há necessidade de ser ajustada a SQTXT'(x) pelos métodos propostos neste trabalho, pois, esta pode ser obtida pela seguin te expressão:

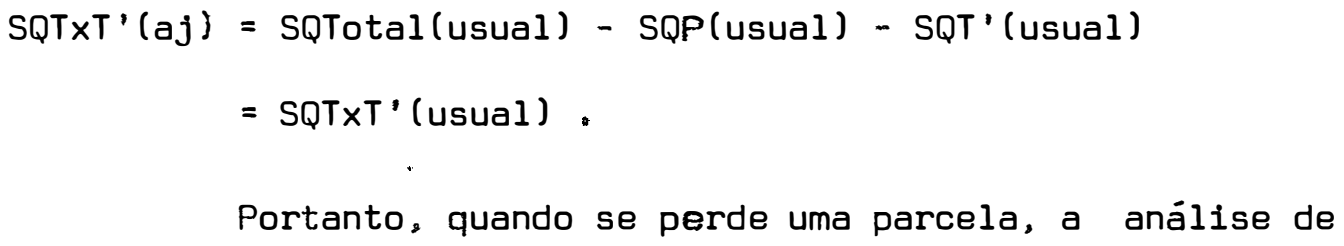
das estimativas das subparcelas perdidas.

As estimativas de variâncias das estimativas de contrastes entre duas médias de tratamentos, são calculadas através das fórmulas apresentadas em 5.7.4, para aplicação dos Testes de Significảncia.

5.7.4 - Estimativas de variâncias das estimativas de contrastes entre duas mëdias de tratamentos

5.7.4.1 - Caso de duas mëdias de tratamentos $T$, on de um deles perdeu duas parcelas

$$
\hat{V}\left(\hat{m}_{i}-\hat{m}_{1},\right)=\left[\frac{1}{2 j}+\frac{1}{2(j-1)}\right] \quad \operatorname{QMR}(a)
$$


5.7.4.2 - Caso de duas médias de tratamentos $T^{\prime}$, on de cada um perdeu uma subparcela

$$
V\left(\tilde{m}_{k_{1}}^{\prime}-\tilde{m}_{k_{2}}^{\prime}\right)=\frac{2}{I J}\left[1+\frac{1}{I(J-1)}\right] \operatorname{DMR}(b)
$$

5.7.4.3 - Caso de duas médias de tratamentos $T^{\prime}$, dentro de um mesmo nível de $T$, onde cada um perdeu uma subparcela

$$
\nabla\left[\left(\tilde{m}_{k 1}^{\prime}-\dot{m}_{k 2}^{\prime}\right) / T_{i}\right]=\frac{2}{J-1} \operatorname{OMR}(b)
$$

Verifica-se que a estimativa da variāncia em 5.7.4.3 é concordante com a apresentada por HADDAD (1978).

5.8 - Caso de Uma Parcela Perdida Composta de k Subparcelas

5.8.1 - Förmulas para estimativas de $k$ subparcelas perdidas $\left(x_{1}, x_{2}, \ldots, x_{k}\right)$ e suas novas estimativas $\left(y_{1}\right.$. $y_{2}, \ldots, y_{k}, z_{1}, z_{2}, \ldots, z_{k}$ ) para utilização do método do resíduo condicional

$$
\begin{aligned}
& x_{1}=\frac{T_{i} T_{k_{1}}^{\prime}}{J-1} \\
& x_{2}=\frac{T_{i} T_{k_{2}}^{\prime}}{J-1} \\
& \ldots . \ldots \\
& x_{k}=\frac{T_{1} T_{k k}^{\prime}}{J-1}
\end{aligned}
$$


68.

$$
\begin{aligned}
& y_{1}=\frac{(J-1)\left(K T_{k_{1}}-G\right)+(I J-1) T_{i}}{K(I J-1)(J-1)} \\
& y_{2}=\frac{(J-I)\left(K T_{K_{2}}^{\prime}-G\right)+(I J-I) T_{i}}{K(I J-I)(J-I)} \\
& y_{k}=\frac{(J-1)\left(k T_{k k}^{\prime}-G\right)+(I J-I) T_{i}}{K(I J-I)(J-I)} \\
& z_{1}=z_{2}=\ldots=z_{k}=\frac{T_{i}}{k(J-1)}
\end{aligned}
$$

5.8 .2 - Correção "U" para a soma de quadrados da interação $\operatorname{TXT}^{\prime}(\mathrm{x})$

A expressão da correção "U" para a soma de quadrados da interação $T_{X} T^{\prime}(x)$ é dada por:

$$
U=\frac{I J-1}{I J} \sum_{k}\left(x_{k}-y_{k}\right)^{2}
$$

Examinando-se esta fórmula, verifica-se que a correção "U" será sempre positiva, portanto, a $S Q T \times T^{\prime}(x)$ estará sempre superestimada, como afirmaram ANDERSON (1946), PINHO (1973), HADDAD (1978) \& PIMENTEL GOMES (1978).

5.8 .3 - Anālise de variância, para o caso de uma parcela perdida composta de $k$ subparcelas

Com os dados do experimento A (Tabela 12), procedeu-se à análise de varłâncla, cujos resultados são apresentados na Tä 
bela 11 .

Tabela 11 - Anālise de variāncia do experimento A, com somas de qua drados (SQ) sem correção e corrigidas [SQ(c)] conforme o proposto neste trabalho, quando foram perdidas quatro subparcelas $\left(x_{531}, x_{532}, x_{533}, x_{534}\right)$ as quais constituiam uma parcela.

\begin{tabular}{lcccc}
\hline Fonte de Variação & G.L. & S.Q. & S.Q. (c) & Q.M. \\
\hline Tratamentos T & 4 & 22,4480 & 22,4480 & $5,6120^{*}$ \\
Residuo (a) & 9 & 13,6079 & 13,6079 & 1,5120 \\
\hline Parcelas & 13 & 36,0559 & 36,0559 & \\
\hline Tratamentos T' & 3 & 17,7281 & 15,1205 & $5,0402 \mathrm{~ns}$ \\
Interação TxT' & 12 & 15,5008 & 14,4015 & $1,2001 \mathrm{~ns}$ \\
Resíduo (b) & 26 & 46,1004 & 46,1004 & 1,7731 \\
\hline Total & & & 111,6783 & \\
\hline
\end{tabular}

Analisando-se a Tabela 1l, percebe-se que as discussões referentes à Tabela 10 são válidas para esta tabela.

As estimativas de variâncias das estimativas de contrastes entre duas médias de tratamentos, são calculadas através das förmulas apresentadas em 5.8.4, para aplicação de Testes de Significāncia. 
5.8.4 - Estimativas de variâncias das estimativas de contrastes entre duas médias de tratamentos

5.3.4.1 - Caso de duas médias de tratamentos T, onde um deles perdeu $k$ subparcelas numa mes ma parcela

$$
O\left(\hat{m}_{i}-\hat{m}_{i}\right)=\left[\frac{1}{J K}+\frac{1}{K(J-1)}\right] \operatorname{OMR}(a)
$$

5.3.4.2 - Caso de duas médias de tratamentos $T^{\prime}$, onde cada um perdeu uma subparcela

$$
V\left(\hat{m}_{k_{1}}-\hat{m}_{k_{2}}^{\prime}\right)=\frac{2}{I J}\left[1+\frac{1}{I(J-1)}\right] \operatorname{QMR}(b)
$$

5.3.4.3 - Caso de duas médias de tratamentos $T^{\prime}$, dentro de um mesmo nível de $T$, onde cada um perdeu uma subparcela

$$
\nabla\left[\left(\hat{m}_{k_{1}}-\hat{m}_{k_{2}}\right) / T_{i}\right]=\frac{2}{J-1} \operatorname{OMR}(b)
$$

Verifica-se que a estimativa da variância em 5.8.4.2

e 5.8.4.3, são as mesmas encontradas em 5.7 .4 .2 e 5.7.4.3. 
71.

\section{CONCLUSÕES}

Com base nos resultados e discussão, podem ser tiradas, para o delineamento inteiramente casualizado com parcelas subdivididas onde ocorreram observações perdidas, as seguintes conclusões:

6.l - A primeira parte da análise de variância deve ser feita de maneira usual, considerando-se um delineamento inteiramente casuali zado com $k$ amostras por parcela.

6.2 - A segunda parte da análise de variância apresentou somente a SQR(b) corretamente estimada, pois, as estimativas das subparcelas perdidas oferecem uma contribuição nula para essa soma de quadrados. 
6.3 - A soma de quadrados da interação TxT', calculada com as es timativas das subparcelas perdidas, estará sempre superestimada.

6.4 - A soma de quadrados de tratamentos T', calculada com as es timativas das subparcelas perdidas, poderá estar super ou subestima da.

6.5 - A soma de quadrados da interação $T \times T^{\prime}$ ajustada pode ser ob tida pela utilização da correção "U", ao invés do método do resíduo condicional de R.A. Fisher, que é mais trabalhoso.

6.6 - As expressões das correções "U" para as somas de quadrados da interação $T_{X} T^{\prime}$, serão sempre positivas, indicando que as $\operatorname{SQTX}^{\prime}(x)$ estarão sempre superestimadas.

6.7 - Nos casos onde foram perdidas uma ou duas subparcelas que não constituíam uma parcela qualquer, para se obterem as somas de quadrados de tratamentos $T^{\prime}$ ajustadas, não serä necessário utilizar o método do resíduo condicional, pois,podem ser obtidas pela seguin te expressão:

$$
\begin{aligned}
\operatorname{SQT}^{\prime}(a j)=\operatorname{SQTotal}(\text { usual }) & -\operatorname{SQP}(\text { usual })-\operatorname{SQT\times T}(\operatorname{aj})- \\
& -\operatorname{SQR}_{1}=
\end{aligned}
$$

6.8 - Quando há perda de uma parcela, constituída de $k \geq 2$ subparcelas, a análise de variāncia pode ser feita de maneira usual, ou seja, sem os cálculos das estimativas das subparcelas perdidas, as 
quais compõem uma parcela.

6.9 - As estimativas de variància das estimativas de contrastes entre duas médias de tratamentos $T$, são obtidas de maneira usual, isto é, considerando-se número diferente de repetições.

6.10 - As estimativas de variància das estimativas de contrastes entre duas médias de tratamentos T', são obtidas através de fórmulas para os seguintes casos:

6.10 .1 - Uma subparcela perdida:

a) Caso de duas médias de tratamentos T', onde um deles perdeu uma subparcela:

$\hat{V}\left(\hat{m}_{k}^{\prime}-\hat{m}_{k 1}^{\prime}\right)=\frac{2}{I J}\left[I+\frac{k}{2 I(J-I)(K-I)}\right] \operatorname{QMR}(b)$,

b) Caso de duas médias de tratamentos T', dentro de um mesmo nível do tratamento $T$, onde um deles perdeu uma subparcela:

$v\left[\left(\hat{m}_{k}^{\prime}-\hat{m}_{k 1}^{\prime}\right) / T_{i}\right]=\frac{2}{J}\left[1+\frac{k}{2(J-1)(K-1)}\right] \operatorname{OMR}(b)$.

6.10.2 - Duas subparcelas perdidas numa mesma parcela composta de $k>2$ subparcelas: 
a) Caso de duas médias de tratamentos T', onde um deles perdeu uma subparcela:

$$
\hat{V}\left(\hat{m}_{k}^{\prime}-\hat{m}_{k I}^{\prime}\right)=\frac{2}{I J}\left[I+\frac{K-I}{2 I(J-1)(K-2)}\right] \text { QMR }(b)
$$

b) Caso de duas médias de tratamentos T', onde ca da um perdeu uma subparcela:

$\hat{V}\left(\hat{m}_{k 1}^{\prime}-\hat{m}_{k 2}^{\prime}\right)=\frac{2}{I J}\left[1+\frac{1}{I(J-I)}\right] \quad \operatorname{QMR}(b) ;$

c) Caso de duas médias de tratamentos T', dentro de um mesmo nível de tratamento $T$, onde um deles perdeu uma subparcela:

$$
\nabla\left[\left(\hat{m}_{k}^{\prime}-\hat{m}_{k 1}^{\prime}\right) / T_{i}\right]=\frac{2}{J}\left[1+\frac{K-1}{2(J-1)(K-2)}\right] \operatorname{QMR}(b)
$$

d) Caso de duas médias de tratamentos T', dentro de um mesmo nível de tratamento $T$, onde cada um perdeu uma subparcela:

$\nabla\left[\left(\hat{m}_{k_{1}}^{\prime}-\hat{m}_{k_{2}}^{\prime}\right) / T_{i}\right]=\frac{2}{J-1} \operatorname{QMR}(b)$.

6.10 .3 - Duas subparcelas perdidas que receberam tratamentos $T$ e T' diferentes: 
a) Caso de duas médias de tratamentos T', onde um deles perdeu uma subparcela:

$$
V\left(\hat{m}_{k}^{\prime}-\hat{m}_{k 1}^{\prime}\right)=\frac{2}{I J}\left[1+\frac{k}{2 I(J-I)(K-I)}\right] \quad Q M R(b)
$$

b) Caso de duas médias de tratamentos T', onde ca da um perdeu uma subparcela:

$$
\nabla\left(\hat{m}_{k 1}^{\prime}-\hat{m}_{k 2}^{\prime}\right)=\frac{2}{I J}\left[1+\frac{K}{I(J-1)(K-1)}\right] \quad \operatorname{QMR}(b)
$$

c) Caso de duas médias de tratamentos T', dentro de um mesmo nível de tratamento $T$, onde um deles perdeu uma subparcela:

$$
\hat{V}\left[\left(\hat{m}_{k}^{\prime}-\hat{m}_{k 1}^{\prime}\right) / T i\right]=\frac{2}{J}\left[1+\frac{K}{2(J-1)(K-1)}\right] \quad Q M R(b) .
$$

6.10 .4 - Duas subparcelas perdidas que receberam tratamentos $T$ diferentes e mesmo tratamento $T^{\prime}$ :

a) Caso de duas médias de tratamentos T', onde um deles perdeu duas subparcelas:

$$
\nabla\left(\hat{m}_{k}^{\prime}-\hat{m}_{k 1}^{\prime}\right)=\frac{2}{I J}\left[1+\frac{k}{I(J-1)(k-1)}\right] \quad \operatorname{QMR}(b) ;
$$


b) Caso de duas médias de tratamentos T', dentro de um mesmo nível de tratamento $T$, onde um deIes perdeu uma subparcela:

$$
\hat{V}\left[\left(\tilde{m}_{k}^{\prime}-\tilde{m}_{k 1}^{\prime}\right) / T_{i}\right]=\frac{2}{J}\left[1+\frac{k}{2(J-1)(K-1)}\right] \text { QMR }(b) .
$$

6.10 .5 - Duas subparcelas perdidas que receberam mesmo tra tamento $T$ e tratamentos $T$ ' diferentes:

a) Caso de duas médias de tratamentos T', onde um deles perdeu uma subparcela:

$$
\begin{aligned}
& V\left(\hat{m}_{k}^{\prime}-\hat{m}_{k l}^{\prime}\right)=\frac{2}{I J}\left\{1+\frac{K(J-I)(K-I)}{2 I\left[(J-1)^{2}(K-I)^{2}-I\right]}\right\} \operatorname{QMR}(b) ; \\
& \nabla\left(\hat{m}_{k_{1}}^{\prime}-\hat{m}_{k_{2}}^{\prime}\right)=\frac{2}{I J}\left\{1+\frac{K}{I[(J-1)(K-1)-I]}\right\} \quad Q M R(b) ;
\end{aligned}
$$

$$
\nabla\left[\left(\hat{m}_{k}^{\prime}-\hat{m}_{k 1}^{\prime}\right) / T_{i}\right]=\frac{2}{J}\left\{1+\frac{K(J-1)(K-1)}{2\left[(J-1)^{2}(K-1)^{2}-1\right]}\right\} \text { QMR }(b)
$$


d) Caso de duas médias de tratamentos T', dentro de um mesmo nível de tratamento $T$, onde cada um perdeu uma subparcela:

$$
\nabla\left[\left(\hat{m}_{k_{1}}^{\prime}-\hat{m}_{k_{2}}^{\prime}\right) / T_{i}\right]=\frac{2}{J}\left[1+\frac{K}{(J-1)(K-1)-1}\right] \operatorname{QMR}(b) .
$$

6.10 .6 - Duas subparcelas perdidas que receberam mesmos tra tamentos $T$ e $T^{\prime}$ :

a) Caso de duas médias de tratamentos $T^{\prime}$, onde um deles perdeu duas subparcelas:

$$
V\left(\hat{m}_{k}^{\prime}-\hat{m}_{k 12}^{\prime}\right)=\frac{2}{I J}\left[1+\frac{K}{I(J-2)(K-1)}\right] \operatorname{QMR}(b) ;
$$

b) Caso de duas médias de tratamentos T', dentro de um mesmo nivel de tratamento $T$, onde um deles perdeu duas subparcelas:

$$
Q\left[\left(\hat{m}_{k}^{\prime}-\hat{m}_{k_{12}}^{z}\right) / T_{i}\right]=\frac{2}{J}\left[1+\frac{k}{(J-2)(K-1)}\right] \operatorname{QMR}(b) .
$$

6.10.7 - Uma parcela perdida composta de $k \geq 2$ subparcelas:

a) Caso de duas médias de tratamentos $T^{\prime}$, onde ca da um perdeu uma subparcele: 


$$
\begin{aligned}
& V\left(\hat{m}_{k_{1}}^{\prime}-\hat{m}_{k_{2}}^{\prime}\right)=\frac{2}{I J}\left[1+\frac{1}{I(J-1)}\right] \text { QMR(b); } \\
& \text { b) Caso de duas médias de tratamentos } T^{\prime} \text {, dentro } \\
& \text { de um mesmo nível de tratamento } T \text {, onde cada um } \\
& \text { perdeu uma subparcela: }
\end{aligned}
$$

$$
\nabla\left[\left(\tilde{m}_{k 1}^{\prime}-\tilde{m}_{k 2}^{\prime}\right) / T_{i}\right]=\frac{2}{J-1} \operatorname{OMR}(b)
$$

6.11 - A maneira mais prática para a correção das somas de quadra dos é através da utilização das correções "U". 


\section{SUMMARY}

The present study is concerned with the determination of formulae to estimate missing subplots, correction factors " $U$ " for interaction TXT' sum of squares and contrasts variances estimates between treatment means in a completely randomized spli-pplot design. It was considered two cases: the case where one or two subplots were missed and the case of a missed whole plot containing $k \geq 2$ subplots.

The missed subplots estimates were determined by minimizing the error sum of squares (b) [SSR(b)]. The correction factors "U" were determined through the conditional error method due to R.A. Fisher.

The adjusted interaction TXT' sum of squares were determined by the conditional error method and by using the correction 
80.

factors "U".

It was shown for the case of one or two missed subplots that the adjusted treatment $T^{0}$ sum of squares [SST'(adj)] can be obtained by

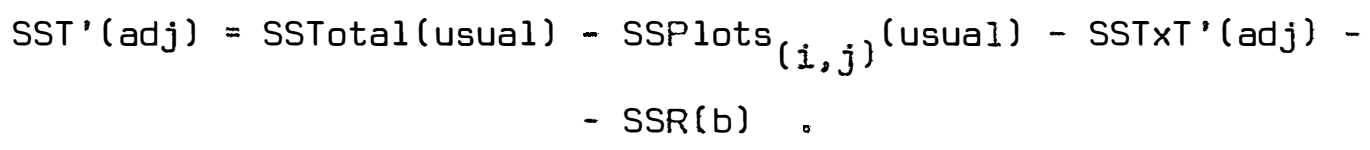

The estimated linear functions of contrasts variances between treatment means were also determined.

For the case of a missed plot containing $k \geq 2$ subplots it was shown that the interaction $T \times T^{\prime}$ and treatment $T$ ' adjusted sum of squares are obtained as in the usual way, that is, by ignoring the estimated missed subplots. 
81.

\section{LITERATURA CITADA}

ALLAN, F。E, e J。WISHART, 1930. A method of estimating the yield of a missing plot in field experimental work. Jour. Agr. Sci., 20: $399-406$.

ANDERSON, R.L。, 1946. Missing-plot techiniques, Biometrics, Carolina do Norte, 2: 41-57.

CAMPOS, H., 1964. Estudo sobre a análise de experimentos com parce las perdidas. Piracicaba, ESALQ/USP, 84 p. (Tese de Doutoramento).

COCHRAN, W.G. e G.M. COX, 1976. Diseños experimentales. 1\% edição, 4: reimpressão, México, Editorial Trillas, $661 \mathrm{p}$.

FREITAS, A.R., 1977. Ensaios realizados pelo Instituto de Pesquisas IRI, em Matão-SP. Seminário apresentado ao Curso de Pós-Graduação em Experimentação e Estatística. Piracicaba, ESALQ/USP, 25 p . 
FREITAS, A。R : I.R. NOGUEIRA \& M.G. FRANCO, 1978。 Ensaios em vasos com leguminosas num delineamento em "split-plot" onde ocorreu per da de parcela. Trabalho apresentado na Reunião Comemorativa dos Noventa Anos do Instituto Agronômico de Campinas. I.A.C., São Paulo, $14 \mathrm{p}$.

HADOAO, M.L., 1978. Estudo sobre parcela perdida em delineamentos em parcelas subdivididas. Piracicaba, ESALQ/USP, 53 p. (Dissertação de Mestrado).

PIMENTEL GOMES, F。, 1978. Curso de Estatistica Experimental. 8: edição, São Paulo, Livraria Nobel, 430 p.

PINHO, S.Z., 1973. Observações perdidas em delineamentos em parcelas subdivididas e parcelas subsubdivididas. Botucatu, FCMBB/ UNESP, 173 p. (Tese de Doutoramento)。

PINHO, S.Z , 1975. Estudo dos componentes de variância nos experimentos em parcelas subdivididas com uma observação perdida. Piracicaba. ESALQ/USP, 45 p. (Dissertação de Mestrado).

STEEL, R.G. e J.H. TORRIE, 1960. Principles and procedures of statistics. Londres, McGraw-Hill, $481 \mathrm{p}$.

YATES, F., 1933. The analysis of replicated experiments when the field results are incomplete. Emp. Jour.Exp.Agr.., 1: 129-142. 
83.

9. APENDICE

DADOS ORIGINAIS DOS EXPERIMENTOS APRESENTADOS COMO EXEMPLOS 


\section{EXPERIMENTO A:}

Tabela 12 - Brix de frutos de 5 variedades de mangueira, colhidos de 3 pés por variedade, e de cada pé se colheram 4 frutos, um de cada um dos pontos cardeais.

\begin{tabular}{|c|c|c|c|c|}
\hline \multirow{2}{*}{ VARIEDADES } & \multicolumn{4}{|c|}{ PONTOS CARDEAIS } \\
\hline & Norte & Sul & Leste & Deste \\
\hline \multirow{3}{*}{ Carlota } & 18,0 & 17,1 & 17,6 & 17,6 \\
\hline & 17,5 & 18,8 & 18,1 & 17,2 \\
\hline & 17,8 & 16,9 & 17,6 & 16,5 \\
\hline \multirow{3}{*}{ Extrema } & 16,3 & 15,9 & 16,5 & 18,3 \\
\hline & 16,6 & 14,3 & 16,3 & 17,5 \\
\hline & 15,0 & 14,0 & 15,9 & 15,2 \\
\hline \multirow{3}{*}{ Oliveira } & 16,0 & 16,2 & 17.9 & 16,1 \\
\hline & 19,5 & 14.9 & 15,0 & 15,3 \\
\hline & 16,3 & 16.4 & 16,0 & $16: 4$ \\
\hline \multirow{3}{*}{ Bourbon } & 16,6 & 15,2 & 14,2 & 155 \\
\hline & 15,9 & 13,2 & 18,0 & 17,3 \\
\hline & 17,5 & 15,8 & 16,7 & 18,4 \\
\hline \multirow{3}{*}{ Imperial } & 18,9 & 18,6 & 15,3 & 17,0 \\
\hline & 18,5 & 13,7 & 18,2 & 18,3 \\
\hline & 21,5 & 16,4 & 18,3 & 16,6 \\
\hline
\end{tabular}

FONTE: Curso de Estatística Experimental. PIMENTEL GOMES, 1978. 


\section{EXPERIMENTO B:}

Tabela 13 - Dados de produção ( $t / h a)$ de cana-de-açúcar, variedade CB-43-5, obtidos de 2 sistemas de plantio nos dois primeiros cortes (cana planta e cana soca).

\begin{tabular}{|c|c|c|}
\hline \multirow{2}{*}{ SISTEMAS DE PLANTIO } & \multicolumn{2}{|c|}{$C O R T E S$} \\
\hline & Cana Planta & Cana Soca \\
\hline \multirow{10}{*}{ Sulcos Simples } & 92,9 & 84,5 \\
\hline & 128,6 & 86,7 \\
\hline & 121,7 & 84,5 \\
\hline & 122,8 & 77,0 \\
\hline & 118,1 & 88,1 \\
\hline & 115,7 & 82,4 \\
\hline & 121,4 & 84,0 \\
\hline & 126,9 & 88,8 \\
\hline & 118,1 & 85,7 \\
\hline & 122,4 & 78,8 \\
\hline \multirow{10}{*}{ Sulcos Duplos } & 122,5 & 84,5 \\
\hline & 110,0 & 85,0 \\
\hline & 115,0 & 85,5 \\
\hline & 125,0 & 88,0 \\
\hline & 105,0 & 86,7 \\
\hline & 110,0 & 80,7 \\
\hline & 115,0 & 88,3 \\
\hline & 105,0 & 89,3 \\
\hline & 108,5 & 94,3 \\
\hline & 118,3 & 90,0 \\
\hline
\end{tabular}

FONTE: Superintendência Geral do PLANALSUCAR em Piracicaba (SP).

Obs.: Espaçamento entre sulcos simples: 1,40 m.

Espaçamento entre sulcos duplos: $0,60 \mathrm{~m} \times 1,40 \mathrm{~m}$." 